\title{
Dual Passband Dichroic Plate for X-Band
}

\author{
T. Y. Otoshi and M. M. Franco \\ Radio Frequency and Microwave Subsystems Section
}

\begin{abstract}
A need arose in the DSN for a dichroic plate that would simultaneously pass two desired frequency bands in the $X$-band region. In addition, the plate must be totally reflective for $S$-band frequencies. This article describes a dielectrically loaded dichroic plate that was developed to meet this need. The unique microwave properties that the new dichroic plate had to possess were (1) insertion losses of less than $0.04 \mathrm{~dB}$ for $X$-band uplink frequencies centered at $7167 \mathrm{MHz}$ and for $X$-band downlink frequencies centered at about $8425 \mathrm{MHz} ;(2)$ insertion losses that met the low-loss requirements at 30-deg incidence angle simultaneously for both parallel and perpendicular polarizations; (3) total reflectivity at S-band frequencies; and (4) ability to maintain these electrical characteristics while passing $100 \mathrm{~kW}$ of $\mathrm{CW}$ power at X-band or while reflecting $100 \mathrm{~kW}$ of $\mathrm{CW}$ power at S-band. The dual-passband dichroic plate that was developed to meet the technical requirements is a thick metallic plate having an array of periodic round holes filled with Teflon plugs. Test results on an experimental prototype plate indicate that it is technically possible to design a dielectrically filled dichroic plate that meets all of the technical requirements.
\end{abstract}

\section{Introduction}

A need arose in the DSN to expand the antenna optics capabilities to include operation at X-band uplink frequencies in addition to maintaining current simultaneous operations at $\mathrm{X}$-band downlink and S-band uplink and downlink frequencies. Although several options are available for converting the present DSN antenna systems to include X-band uplink operation, most of the options are expensive to develop and implement.

A relatively simple option was suggested by $R$. Clauss of the JPL TDA Technology Development Office. His suggestion involved the development of a dichroic plate of the same type currently being used in the DSN, with the exception that the array of holes in the dichroic plate be filled with Teflon material. It was pointed out by Clauss that, based on a simple analysis, the proper choice of hole diameter, dielectric material, and plate thickness would result in a dichroic plate that would have passbands at the desired X-band uplink and $\mathrm{X}$-band downlink center frequencies. It was also pointed out by Clauss that a dielectric loaded plate would permit hole diameters and hole-to-hole spacings to be smaller than those of an equivalent air-filled dichroic plate. If a design of an equivalent dual passband air-filled dichroic plate were attempted, a cursory analysis would indicate that grating lobes would appear at downlink frequencies when the plate is used at incidence angles in the region of $30 \mathrm{deg}$. A plate with closely packed, dielectric filled holes would tend to have fewer grating lobe problems at the higher incidence angles. 
The approach of dielectrically filling the holes appeared to be technically promising and relatively easy to develop and implement. However, it was not certain that a more rigorous theoretical analysis would lead to the same conclusion as the simple analysis when taking into account multi-mode waveguide, incidence angle, and polarization considerations. Experimental measurements needed to be performed in order to accurately determine noise temperature contributions of the dielectrically loaded plate. Also, antenna patterns needed to be taken to verify the absence of potential grating lobes and the absence of unknown losses such as those that might be caused by surface wave propagation. It became necessary to initiate a theoretical study and an experimental verification program for dielectrically loaded plates.

A program was initiated to design, fabricate, and test a model plate to demonstrate that a dielectrically loaded dichroic plate could be developed to meet the specified technical requirements. Section II of this article presents descriptions of the dichroic plates that were designed and fabricated and additionally presents corresponding theoretical data. Section III presents results of insertion loss, noise temperature, and antenna pattern measurements. The project status is discussed in Section IV, and a summary and conclusions are presented in Section V.

\section{Dichroic Plate Designs and Theoretical Data}

Figure 1 shows the geometry of the dichroic plate. The angle theta is the angle of incidence of the incoming plane wave, and phi is the spherical coordinate azimuth angle defined with respect to the hole pattern geometry shown in Fig. 1. All dichroic plates currently used in the DSN are plates with air dielectric holes. The new proposed plates will have holes that are filled with a solid, low-loss dielectric material such as Teflon.

The requirements for new generation dichroic plates are that they be transparent to both X-band uplink and X-band downlink frequencies and simultaneously be (nearly 100 percent) reflective to S-band uplink and downlink frequencies. The dichroic plate must be able to transmit $100-\mathrm{kW} \mathrm{X-band}$ uplink power without arcing. The following are the microwave technical requirements that the plate must meet:

\section{$\mathrm{X}$-band uplink:}

$7145-7190 \mathrm{MHz}$ (insertion loss $<0.04 \mathrm{~dB}$ )

$7145-7235 \mathrm{MHz}$ (insertion loss $<0.10 \mathrm{~dB}$ )

$\mathrm{X}$-band downlink:

$8400-8450 \mathrm{MHz}$ (insertion loss $<0.04 \mathrm{~dB}$ )

(dissipative loss $<0.02 \mathrm{~dB}$ )
$8400-8500 \mathrm{MHz}$ (insertion loss $<0.10 \mathrm{~dB}$ )

(dissipative loss $<0.04 \mathrm{~dB}$ )

S-band uplink and downlink:

$2090-2320 \mathrm{MHz}$ (reflective insertion loss $>45 \mathrm{~dB}$ )

The plate must have these microwave properties for both perpendicular and parallel polarizations (Fig. 1) at 30-deg incidence angle at a specified phi angle orientation. The requirement for optimization at these particular theta and phi angles was dictated by the antenna optical system [1] that the plate would be used in. It was also a goal to find a final plate design that would give the best performance over all incidence angles from $15 \mathrm{deg}$ to $45 \mathrm{deg}$, and a particular phi angle plus and minus 5 deg.

A computer program based on equations derived by Chen [2], [3] was used to design the dielectrically loaded dichroic plate. The original computer program, which was written by Chen for an air dielectric hole medium, was modified by Otoshi to allow the medium in the hole to have any relative permittivity and loss tangent value. After many computer runs, data analyses, and numerous trial designs, it was concluded that all of the requirements could not be met simultaneously for both polarizations and both X-band passbands at 30-deg incidence angle if the aperture is restricted to a round hole. The lack of identical plate properties for both polarizations is a serious problem because it gives rise to elliptical polarization output when the incident wave is circularly polarized.

The only possible method for correcting this polarization problem is to go to a two-diameter (elliptical or two-cutoff wavelength waveguide) design of the type currently being used in the DSN [4]. However, a decision was made to try out the dielectrically filled round-hole design first, because a roundhole design could be handled by an existing computer program. A two-diameter loaded plate that is filled with dielectric plugs is also more expensive to fabricate than a dichroic plate with round holes.

Computer runs made of round-hole dichroic plates showed that if Teflon is used for the dielectric plug material, then the dielectric constant needs to be known to about 5 percent accuracy. The plates are expensive to fabricate. Computer runs with the Chen-Otoshi program showed that if the dielectric constant is in error by more than 5 percent, the plates will not have the passbands at the correct center frequencies. At the start of this project, the values of Teflon were not known well. References [5]- [7] give values for the dielectric constant of Teflon that varied from 2.01 to 2.08 over the X-band region.

A study program was initiated to determine the dielectric constant of the particular Teflon stock actually used in fabri- 
cating the plugs for the plates. After studying various methods of measurement of dielectric constants, the Automatic Network Analyzer method [6], [7] was selected. The HP 8510A Automatic Network Analyzer equipment was readily available and data reduction software could be (and was) written inhouse. The Teflon samples were made oversize by about 0.002 inch and press-fitted into WR 112 waveguides. For the pressfitted waveguide Teflon samples made from the same stock material to be used to fabricate plugs for the dichroic plates, the measured dielectric constant was $2.04 \pm 0.01$ over the frequency region of 7.0 to $9.0 \mathrm{GHz}$. A value of 2.04 was used to design the plates. If a lower than actual dielectric constant were used to design the plate, the result would be a plate thickness that is too large. However, this situation might not be serious because it might be possible to later trim the plate thickness to cause the minimum insertion point to shift to the desired center frequency.

A total of three dichroic plates having Teflon plugs were fabricated. The plates had numerous (more than 1000) holes that were closely packed. To avoid tolerance buildup of the hole locations, the plates were fabricated with the aid of a computer-programmed milling machine. All of the coordinates for the holes were computer-programmed, and the coordinate of each hole was kept to within a 0.002 -inch tolerance. The material for the plate was 6061-T4 aluminum, and the holes were filled with Teflon plugs made from Teflon rods conforming to Materials Specification MIL-P-19468. The method for holding the plugs in the holes was to make their diameters 0.005 -inch oversize, shrink the plugs in liquid nitrogen, drop them into the holes, and allow the plugs to expand at room temperature. The plates were then machined to the proper thickness.

Figure 2 shows one of the fabricated plates with its holes filled with Teflon plugs. The theoretical performance of this plate, labeled Plate \#1 for convenience, is shown in Fig. 3 for the ta $=30 \mathrm{deg}$ for parallel and perpendicular polarizations. The theoretical values were based on a Teflon dielectric constant of 2.04 and a handbook loss tangent value of 0.00015 [5]. It is sufficient to state that the theoretical curves for Plate \#1 show two passbands, a peak-to-peak insertion loss variation of about $2 \mathrm{~dB}$ over the band, and a resonance (due to higher-order TM modes) for parallel polarization. The range of the $\mathrm{Y}$-axis is purposely made large to permit comparison with an insertion loss plot for an air-filled dichroic plate, which will be discussed later. In order to show low insertion loss values in the passband in more detail, the theoretical values are given in Table 1 along with the plate dimensions. It should be restated that Plate \#1 is a round-hole design, and that a compromise had to be made to give the best performance for X-band downlink for both polarizations and to give an acceptable performance for $\mathrm{X}$-band uplink frequencies.
Plate \#2 differed from Plate \#1 in that the plate was not as thick and the holes were drilled at a slant angle of $30 \mathrm{deg}$ from the normal for the plate. The reason for drilling the holes 30 deg from normal was that since the plate is to be used for a 30-deg angle of incidence in the actual application, the direction of propagation of the incident ray would be exactly in line with the longitudinal axis of the holes. It was expected that the impedance match would be better and hence would make the passband of the plate broader and also less sensitive to changes of incidence angles in the region of $30 \mathrm{deg}$.

Plate \#3 is the third plate having Teflon plugs as the medium filling the holes of the plate. This plate was originally the round-hole dichroic plate that was designed by Potter and is described in [4]. Although it was known that, when dielectrically loaded, this plate would not have the passbands at the desired uplink and downlink X-band frequencies, this plate was readily available and provided an opportunity to immediately verify the computer program used to design other dielectric filled dichroic plates (Plates \#1 and \#2). Although this plate is labeled Plate \#3 (for convenience), this plate with Teflon plugs was fabricated and tested 2 months before the start of fabrication of Plates \#1 and \#2. Insertion loss measurements on Plate \#3 revealed that theory and experiment were in good agreement to within an accuracy of $\pm 0.3 \mathrm{~dB}$ for the measurement method that was then employed. Based on the initial test results, a decision was made to proceed with the fabrication of Plates $\# 1$ and $\# 2$, which were already designed.

Figure 4 shows an air-filled dichroic plate, labeled Plate \#4, which was used as a comparison standard for verifying the performance and integrity of insertion loss, noise temperature, and antenna pattern measurement test setups. This plate is the original Pyle waveguide dichroic plate, also designed by Potter and described in [4], and is the plate design currently being used in the DSN. To be useful as a comparison and verification standard, however, it was necessary to validate the performance of this plate as well.

Although some data on Plate \#4 was available in lab notebooks and publications [4], there were no broadband theoretical or accurately measured insertion data that could be found on this plate. The original computer program used by Potter for the Pyle waveguide aperture also could not be located. Therefore, another approach had to be used to generate theoretical data for the Pyle waveguide dichroic plate. The approach used was to take the design dimensions of the Pyle waveguide on the dichroic plate and find the cutoff wavelengths from the Pyle waveguide curves given in [4]. Then the equivalent TE11 mode circular waveguide diameter was found from these cutoff wavelengths for the applicable polarizations. The perpendicular polarization curve was computed using one round-hole diameter, and the parallel polarization curve was 
computed using the other round-hole diameter. The theoretically calculated curves are shown in Fig. 5. Theoretical insertion loss values in the passband as well as plate dimensions for this plate are tabulated in Table 2. Figures 6 and 7 show experimentally obtained data that are in excellent agreement with the theoretical values. The acquisition of excellent experimental insertion loss data was made possible by making the insertion loss measurements in an anechoic chamber and using an automatic network analyzer. An interesting observation when examining the theoretical curves for the dielectrically filled dichroic plate (Fig. 3) and for the Pyle waveguide plate (Fig. 5) is that the air-filled plate appears to have low insertion losses over a wider frequency band.

All of the plates were fabricated to have the same outer diameter of about 33 inches. Each plate had the same mounting hole patterns to enable interchangeability in the test setups. By having all of the plates the same size, it was possible to make accurate comparisons to a standard reference plate whose test results were already known. It was also possible to make measurements of four plates in the same test setup without altering the setup once the integrity of the setup was verified. Test setup, calibration, and verification took up the most time in the measurement process. Once the test setups were verified, the interchangeability of plates enabled measurements of plates to be made relatively quickly.

Although all four plates were tested thoroughly, in order to keep this article to a reasonable length only the results of Plates \#1 and \#4 will be reported. Results on the other plates will be presented in future publications.

\section{Experimental Results}

\section{A. Insertion Loss Tests}

Low insertion loss in the passband is one of the more important properties that a dichroic plate must have when used in a deep space communications receiving system. Insertion loss includes losses due to both reflection and dissipation. The insertion loss of a plate can be determined from measurements made of the magnitude of the transmission coefficient S21 of the path between the transmit and receive horns with and without the dichroic plate inserted into the path. A block diagram of the test setup used to make insertion loss measurements on a dichroic plate is given in Fig. 8. An outdoor test setup with one of the plates being tested is shown in Fig. 9. Perpendicular-to-parallel polarization changes are made by a 90-deg rotation of the circular-to-rectangular waveguide transitions connected to the transmit and receive horns. Rapid 90 . deg rotations of these waveguide transitions were facilitated through the use of low-loss, low-VSWR circular waveguide rotary joints.
To enable accurate insertion loss measurements to be made over a broad band of frequencies, the HP 8510A automatic network analyzer was used. The procedure was to first make a reference measurement of $S 21$, the transmission coefficient, for the total system without the plate. The total system with. out the plate consists of cables, coaxial-to-rectangular waveguide transitions, waveguide isolators, rectangular-to-circular waveguide transitions, the transmit and receive horns, the freespace propagation path, and the dichroic plate holder. The dichroic plate holder (without the plate) was first tilted at the desired incidence angle with the horns oriented for the desired polarization, and then measurements were made of $S 21$ and recorded as a function of frequency. This first set of measurements provided the reference data. Then the test was repeated with the plate attached to the plate holder. The automatic network analyzer was then used to convert the $S 21$ data into $d B$, and the first (or reference) measurement data were subtracted from the second (or test) measurement data, thus resulting in plate-only S21 data in decibels. Data were taken from $7.0 \mathrm{GHz}$ to $9.0 \mathrm{GHz}$ in $5-\mathrm{MHz}$ steps and stored on diskette files. Insertion group delays of the plates were determined from S21 phase data, which were simultaneously recorded.

Figures 10 and 11 , respectively, show measured insertion losses for perpendicular and parallel polarizations for the dielectrically loaded Plate \#1 when the plate was mounted for a $30-\mathrm{deg}$ incidence angle and $\mathrm{phi}=90 \mathrm{deg}$. The measurements were made in the outdoor setup, rather than in an anechoic chamber, to avoid having to pay the high rental cost ( $\$ 400$ per day) of using the anechoic chamber. In Figs. 10 and 11, frequency markers show the desired uplink and downlink passbands and the approximate regions where the plates were theoretically supposed to have minimum insertion loss as predicted by the Chen-Otoshi program (see Table 1). It can be seen that the experimental and theoretical passbands agreed reasonably well for perpendicular polarization, but the experimental passband was about $55 \mathrm{MHz}$ too high for the parallel polarization configuration. ${ }^{1}$ Other measurements were also made at 0-, 15-, 45-, and 60-deg incidence angles as well as at other phi angles. In order to keep this article to a reasonable length, all test data presented will be restricted to the configuration of an incidence angle of $30 \mathrm{deg}$ and $\mathrm{phi}=90 \mathrm{deg}$, which is the geometry that dichroic plates are in when installed on DSN antennas.

Figures 12 and 13 show the insertion loss of Plate \#4 as measured in the outdoor test setup. This plate was used as the standard to check out the integrity of the outdoor insertion

\footnotetext{
${ }^{1}$ Slightly better agreement between theory and experiment was obtained at a $30-\mathrm{deg}$ incidence angle test configuration where the plate orientation was changed from a phi angle of $90 \mathrm{deg}$ to a phi angle of $0 \mathrm{deg}$.
} 
loss test setup because the insertion loss properties of this plate were known beforehand. Comparisons of the outdoor test setup data (Figs. 12 and 13) with theoretical data in Fig. 5 show that valid insertion loss measurements could be made with the outdoor test setup.

It should be mentioned that for measurements of insertion loss in the outdoor test setup, ripples on the insertion loss as a function of frequency were observed in the test data. These ripples are attributed to multipath signals interacting with the primary desired signal. Proper placement of absorbing material around the horns, frame holder, and flat reflecting surfaces helped reduce the magnitude of multipath ripples. In an outdoor setup such as the one that was used (Fig. 9), it is difficult to totally eliminate the multipath-caused ripples. When a limited number of tests were later made in an anechoic chamber, it was observed that multipath signal-caused ripples on measured insertion loss were almost nonexistent.

The cause of the discrepancy between theory and experiment for dielectrically loaded Plate \#l was originally thought to be an error in the dielectric constant value of 2.04 used to design the plate. However, use of different dielectric constant values ranging from 2.01 to 2.10 through the computer program failed to explain the discrepancy between the theoretical and experimental data for this plate. Hence, an incorrect dielectric constant was ruled out as the possible cause of the discrepancy.

Another cause of the discrepancy between theory and experiment for the dielectrically loaded plate was thought to be poor machining of the surfaces of Plate \#1. Some of the Teflon plugs were recessed from the surface of the plates and some of the ends of the Teflon plugs had prominent machining marks and grooves. This uneven plate surface gave rise to the hypothesis that errors could be generated by unwanted surface waves. The plate surfaces were therefore remachined with an extra-sharp cutting tool. The final plate thickness was 0.017 inch less than the original 1.580 inch. However, the machining of the plate to a fine surface finish did not eliminate the discrepancy between theoretical values (based on the new thickness) and experimental values for the parallel polarization case. It should be pointed out that the plate was not remachined to the new thickness until all other data (noise temperature and antenna patterns) were first taken.

Another possible cause of the discrepancy between theory and experiment was thought to be an insufficient number of Floquet modes used in the Chen-Otoshi program, which generated the theoretical data. Changing the number of Floquet modes from $(\mathrm{p}, \mathrm{q}=11,5)$ to $(\mathrm{p}, \mathrm{q}=51,51)$ did not make any significant difference in the theoretical values.
The only other presently known explanation for the discrepancy between theory and experiment is that the theoretical data were computed on the basis of matching the freespace parallel polarization tangential E-field to 5 TM waveguide modes at the dichroic plate interface. When the plate is dielectrically loaded, the number of TM waveguide modes may have to be increased in order to compute the transmission coefficient more accurately when the plate is tilted at $30 \mathrm{deg}$. Adding more waveguide modes to the existing version of the Chen-Otoshi program turned out to be difficult. At this time, modifications of the Chen-Otoshi program to include additional waveguide modes are still in progress. No definite explanation exists at this time as to the cause of the discrepancy between theoretical and experimental results. The lack of a good theoretical computer program also makes it more difficult to design dielectrically loaded dichroic plates for other frequency bands.

\section{B. Noise Temperature Tests}

Noise temperature measurements were made because insertion loss measurements in the outdoor setup could not give results to accuracies of better than $0.1 \mathrm{~dB}$. A good noise temperature setup, where mismatch errors are small, has the potential for providing dissipative loss information to accuracies of better than $0.1 \mathrm{~K}$, corresponding to a dissipative loss of about $0.002 \mathrm{~dB}$.

The method for determining noise temperature of the dichroic plate involves the measurement of effective receiver temperature with and without the plate inserted between the horn and sky. The change in measured effective receiver temperature is attributed to the dichroic plate dissipative loss, which includes plate surface resistivity loss, waveguide conductivity loss, and dielectric dissipative loss. The equations and description of the procedure are described elsewhere, ${ }^{2}$ and hence will not be described further here.

Figures 14 and 15 show the test setups used for the noise temperature measurements of the plate for perpendicular and parallel polarizations, respectively. It was necessary to test the plates for both polarizations because the insertion loss test results showed that significant differences existed for the two polarizations. The basic equipment required for this measurement technique includes a calibrated low-noise horn of known noise temperature, a low-noise maser, a follow-up receiver, noise power measurement equipment, and an ambient load to place over the horn aperture. An attempt was made to rereflect the power reflected by the dichroic plate back up to

\footnotetext{
2 "X-Band Traveling Wave Maser Group Block IIA," Part I of II, Technical Manual Overhaul Instructions (internal document), Jet Propulsion Laboratory, Pasadena, California, pp. 113-119, August 1, 1983.
} 
the sky. This was achieved by placing a secondary reflector plate parallel to the dichroic plate at a 30-deg angle from the horizontal (see Figs. 14 and 15). This secondary reflector was a solid aluminum sheet with a 33 -inch diameter. Some of the open spaces between the horn aperture and the secondary reflector were covered with metal plates and aluminum foil. Test results later indicated that the 33-inch diameter secondary reflector was inadequate in size and should have been fabricated to have a larger diameter. It is valid to reflect the main reflection lobe from the dichroic plate back up to the sky with the secondary reflector because, in the actual application of the dichroic plate on the large antenna, the reflections from the dichroic plate are re-reflected to the sky by the large main reflector of the Cassegrainian antenna.

Table 3 shows the results of noise temperature tests for Plates \#1 and \#4. Plate \#4 was used as the test setup verification plate because its noise temperature had been previously calibrated. The measured noise temperature value of $1.5 \mathrm{~K}$ in this current setup agrees well with expected values for Plate \#4. The noise temperature of about $4.5 \mathrm{~K}$ for Plate \#1 was higher than anticipated. Theoretically, Plate \#1 should have a noise temperature of about $2.5 \mathrm{~K}$, of which $1.5 \mathrm{~K}$ is due to plate surface resistivity and waveguide conductivity loss while the remaining $1.0 \mathrm{~K}$ is due to Teflon dissipation loss (as theoretically calculated from the Chen-Otoshi program when using a loss tangent of 0.00015 for Teflon). Therefore, the measured noise temperature of the dielectrically loaded plate was about $2.0 \mathrm{~K}$ higher than expected.

It was initially thought that the higher loss of Plate \#1 might be due to the fact that the loss tangent of Teflon is 2 or 3 times higher than the published handbook values. Studies were made later to accurately determine the loss tangent of Teflon. Through the use of two independent measurement techniques, it was established that the Teflon material used for plugs in the dichroic plate had a loss tangent of 0.0002 . When this value of loss tangent is used in the Chen-Otoshi modified computer program, a $1.5-\mathrm{K}$ noise temperature due to dielectric loss is calculated. The two methods used to determine loss tangent were (1) an improved automatic network analyzer measurement technique; and (2) an improved noise temperature measurement method. These two improved methods and associated test results will be discussed in a separate report. It was important to determine the loss of Teflon accurately because Teflon is one of the less expensive readily available materials that have low loss. It was desirable to prevent erroneous conclusions from being drawn based upon the dichroic plate noise temperature measurements. It would be a serious mistake to conclude prematurely that dielectrically loaded dichroic plates are not useful because they have undesirably high noise temperatures generated by dielectric losses.
The poor results in Table 3 for Plate \#1 might be explained by the following equations, which are applicable to the test setups shown in Figs. 14 and 15. The equations for system noise temperature for the measurement setups for the configuration with and without the dichroic plate are respectively given as:

$$
\begin{aligned}
\mathrm{T}_{s y s}= & \mathrm{T}_{s k y}+\mathrm{T}_{\text {horn }}+\mathrm{T}_{\text {maser }}+\mathrm{T}_{f r} \\
\mathrm{~T}_{s y s}^{\prime}= & \left(1-\left|\Gamma_{d p}\right|^{2}\right) \mathrm{T}_{s k y}+\mathrm{T}_{d p}+\left|\Gamma_{d p}\right|^{2}\left|\Gamma_{s p}\right|^{2} \mathrm{~T}_{s k y} \\
& +\left|\Gamma_{d p}\right|^{2}\left(1-\left|\Gamma_{s p}\right|^{2}\right) \mathrm{T}_{p g}+\mathrm{T}_{\text {horn }}+\mathrm{T}_{\text {maser }}+\mathrm{T}_{f r}
\end{aligned}
$$

Then

$$
\begin{aligned}
\mathrm{T}_{\text {diff }} & =\mathrm{T}_{s y s}^{\prime}-\mathrm{T}_{s y s} \\
& =\mathrm{T}_{d p}+\left|\Gamma_{d p}\right|^{2}\left(1-\left|\Gamma_{s p}\right|^{2}\right)\left(\mathrm{T}_{p g}-\mathrm{T}_{s k y}\right)
\end{aligned}
$$

where

$$
\begin{aligned}
\mathrm{T}_{s k y}= & \text { sky noise temperature (approximately } 5 \mathrm{~K} \text { ) due to } \\
\text { cosmic background }(2.7 \mathrm{~K}) \text { and the zenith atmo- } & \text { spheric noise temperature (nominally } 2.3 \mathrm{~K} \text { ) at } \\
& \text { X-band, } \mathrm{K}
\end{aligned}
$$

Equation (3) shows that the error in measuring the noise temperature of the dichroic plate will be proportional to the mag. nitude squared of the reflection coefficient of the dichroic plate times the ratio of power not re-reflected by the secondary plate. For example, if at the noise temperature measurement frequency, the insertion loss of a dichroic plate is $0.1 \mathrm{~dB}$, corresponding to a voltage reflection coefficient magnitude 
of 0.15 , and the secondary plate captures and reflects only 90 percent of the power radiated toward it (as a result of reflection from the dichroic plate), then from Eq. (3) the error in measuring the noise temperature of the dichroic plate will be

$$
0.15 \times 0.15 \times(1.0-0.90) \times(290-5)=0.6 \mathrm{~K}
$$

If instead the insertion loss of the plate is $0.5 \mathrm{~dB}$, corresponding to a voltage reflection coefficient magnitude of 0.33 , the error in measurement will be $3.1 \mathrm{~K}$. A study of this type is being made to correlate the insertion loss data measured for Plates \#1 and \#4 to noise temperature errors at the various measurement frequencies and the two polarizations. The results of this study will be reported later.

A good test would have been to cover the lower side of the dichroic plate with a metallic sheet having the same diameter as the dichroic plate. This would simulate a worst-case reflection coefficient of 1.0 from the dichroic plate. All of the power coming from the dichroic-plate reflection-lobe direction could be captured by a properly designed secondary plate, and this captured power could then be redirected towards the sky. According to Eq. (3), and taking into account resistive losses on the reflectors, a measurement of antenna temperature would reveal how effective the secondary plate was and how much noise temperature was due to ground absorption.

\section{Antenna Patterns}

As a final test, antenna patterns were taken of the dichroic plates to verify that there were no unknown effects such as unknown grating lobes, beam shifting, or scattering patterns not predicted by theory. Antenna patterns taken in different planes will give information about scattering losses in three dimensions, whereas insertion loss measurements basically give information only in one propagation direction between the transmit and receive horns.

The measurement of antenna patterns is more laborious and time consuming because, at a single frequency, a pattern must be recorded for $360 \mathrm{deg}$ of angle rotation. There are main E-plane patterns (corresponding to parallel polarization) and main H-plane patterns (corresponding to perpendicular polarization) as well as cross-polarization patterns that have to be taken. Then the same types of patterns have to be taken with the plate rotated 90 deg so that the plate coordinate system is rotated $90 \mathrm{deg}$ with respect to the axis of rotation for pattern recording.

Figure 16 shows the antenna pattern measurement setup for measurement of the horn only in the $60 \mathrm{ft}$ anechoic chamber at the JPL Mesa Facility. The phase center of the horn was aligned with the axis of rotation of the table. The procedure was to measure the pattern of the horn only, and then to measure patterns with the dichroic plate installed in place. Then the dichroic plate assembly was turned over on its side $90 \mathrm{deg}$ and the pattern was again recorded. Figure 17 shows the test configuration where the plate holder and plate are in an upright position similar to that used for insertion loss measurements. In this configuration, the main reflection from the plate will be radiated toward the ceiling of the anechoic chamber and be absorbed. Figure 18 shows the test configuration where the plate is turned over on its side $90 \mathrm{deg}$. In this test configuration, the main reflection lobe will appear in the antenna pattern recording. The plate was mounted for a phi $=90-\mathrm{deg}$ hole pattern orientation angle, and a 30-deg incidence angle position. The rotator angle reads $0 \mathrm{deg}$ and the receive and transmit horns are boresighted to each other. Correct alignment and boresighting of the transmit and receive $\mathrm{X}$-band horns were previously verified with an optical laser beam.

E- and H-plane patterns were taken of the horn only, horn plus plate holder, and horn plus plate holder and plate for both the upright and flopped 90-deg positions. All correspond. ing cross-polarization patterns according to Ludwig definition \#1 [8] were also taken. All of these types of patterns were taken for Plate \#1 at uplink frequencies of $7145 \mathrm{MHz}$ and $7168 \mathrm{MHz}$ and also at downlink frequencies of $8415 \mathrm{MHz}$, $8425 \mathrm{MHz}$, and $8450 \mathrm{MHz}$.

For the DSN plate (Plate \#4), only E- and H-plane patterns at $8415 \mathrm{MHz}$ were taken for verification purposes. The antenna patterns were virtually identical to those given in [4] and, therefore, measured patterns for Plate \#4 will not be presented in this article.

Figures 19 and 20, respectively, show E- and H-plane patterns for the horn alone at a frequency of $7168 \mathrm{MHz}$, which corresponds approximately to the middle of the desired uplink passband. Figures 21 and 22, respectively, show the antenna patterns when dichroic Plate $\# 1$ and the plate holder were installed in the flopped 90-deg position. Figures 23 through 26 show similar sets of patterns taken at the downlink mid. passband frequency of $8425 \mathrm{MHz}$. Patterns taken with the frame assembly upright were virtually the same as the hornalone patterns, with the exception of about $0.2 \mathrm{~dB}$ loss in the peak of the main beam relative to the horn-alone case. Therefore, patterns taken with the dichroic plate holder in the upright position (see Fig. 17) will not be shown. Crosspolarization patterns will not be shown because in all cases the patterns were at least $35 \mathrm{~dB}$ down from the peak of the main beam of the main polarization patterns.

An interesting correlation can be made of antenna pattern data and the insertion loss data given in Figs. 10 and 11. If the 
level of the largest reflection lobe is subtracted from the peak level of the main beam of the horn-only pattern, and the difference of levels is treated as return loss, these data can be converted to insertion loss. The results of this analysis are given in Table 4. Reasonable agreement was obtained between insertion loss values computed from pattern data and insertion loss values ( $\pm 0.1 \mathrm{~dB}$ accuracy) in the outdoor test setup.

One final test was needed to show that Plate \#1 behaves as a solid reflector at $\mathrm{S}$-band frequencies. In order to perform the required test, it was necessary to replace the transmit and receive X-band horns in the anechoic chamber test setup with $S$-band standard gain horns. The particular type of standard gain horn used was a Scientific-Atlanta (Model 12-1.7) standard gain horn that has a WR430 waveguide input and a gain of about $16.4 \mathrm{~dB}$ at $2295 \mathrm{MHz}$. Antenna patterns were made at $2295 \mathrm{MHz}$ with Plate \#1 in the 90-deg flopped position, and then again after the front face of the plate was covered with a thin solid aluminum sheet of the same diameter. No differences were observed in the two patterns. It is safe to conclude that below cutoff, a dielectric loaded plate has properties similar to a perfect reflector at S-band. This conclusion is supported by theoretical data obtained from the Chen-Otoshi computer program.

The antenna pattern tests verified that there was no unusual behavior of the dielectrically loaded dichroic plate such as might be caused by unexpected surface wave radiations or grating lobes. The experimental work generally confirmed most of the theoretical predictions concerning the behavior of dielectrically loaded dichroic plates.

\section{Project Status}

\section{A. Accomplishments}

The following is a list of accomplishments and spinoffs resulting from this project:

(1) Numerous computer programs written in FORTRAN and BASIC for IBM-XT/AT, including:

(a) Waveguide conductivity loss for a circular or rectangular waveguide when the waveguide is filled with any dielectric material;

(b) Below cutoff attenuation for numerous TE and TM modes for a circular or rectangular waveguide of specified length and frequency;

(c) Generation of theoretical S-parameters for a dielectric sheet in either a circular or rectangular waveguide; (d) Derivation of complex permeability and complex permittivity values from measured $S 11$ and $S 21$ on a dielectric-filled waveguide sample;

(e) Improvement of measurements of noise temperature when using the horn-maser Y-factor measurement technique. The improvement occurs in the data reduction that accounts for atmospheric noise temperature changes due to weather condition changes. (This improvement technique might be valuable for future Beam Waveguide system evaluations.);

(f) Antenna computer programs related to dichroic plate measurements such as antenna range loss (Friis transmission loss formula), far-field criteria, and circular and rectangular aperture theoretical antenna gain programs; and

(g) A computer program to compute group delay from network analyzer phase measurements.

(2) Two methods were developed for measuring the permeability and permittivity of materials. The first method involves the measurement of S-parameters of the dielectric sample in a waveguide. The second method involves the measurement of the sample in a waveguide when terminated with a short-circuit. In addition, sig. nificant improvements were made on the current network analyzer technique to accurately determine the loss tangent of low-loss dielectric materials. Heretofore, the network analyzer technique could not be used to accurately determine loss tangents of low-loss dielectric materials.

(3) A new noise temperature measurement technique was developed for the accurate determination of dissipative loss of dielectric materials from noise temperature measurements. This method uses the technique of making the waveguide sample an integer multiple of a half. waveguide wavelength at the test frequency. Substitution of this waveguide sample for an empty waveguide of identical electrical length (already in the system) enables cancellations of waveguide conductivity loss and minimization of mismatch errors.

Other new results obtained on dichroic plates not yet reported include the following:

(1) Good agreement was obtained between theoretical and measured group delays of Plates \#1 and \#4;

(2) Data were taken with various-thickness Teflon sheets placed on one or both sides of dichroic plates. The 
passbands shifted, but as yet the experimental data have not been correlated to theory. The employment of dielectric sheets might be a good way to fine-tune the dichroic plate passband characteristics;

(3) It was found that the insertion losses of a dichroic plate, as measured over a frequency range of 7.0 to 9.0 $\mathrm{GHz}$ with the test setup configurations shown in Figs. 8 and 9 , did not change when the transmit X-band horn having a diameter of 7 inches was replaced by a pyramidal horn whose largest dimension was 5 inches. The multipath ripple magnitude increased for data taken with the smaller horn, but the average measured insertion loss curve (see Figs. 10 and 11) was the same for either transmit horn test setup;

(4) Data obtained on the slant-drilled Teflon-loaded dichroic Plate \#2 described in Section II showed that higher than expected insertion loss occurred for parallel polarization and 30-deg incidence angle at X-band downlink frequencies. The corresponding noise temperatures were also much higher than expected. No beam squint was observed on the antenna patterns for this plate;

(5) Improved techniques for future insertion loss measurements of dichroic plates were learned. It was found that time-consuming calibrations could be skipped if isolators were used. Also, it was learned that a solid reflector should be used to check for leakage if very high insertion values are to be measured, such as for a dichroic plate below cutoff;

(6) Improvements in noise temperature measurements of a dichroic plate can be obtained if a solid plate is placed in front of the dichroic plate. This measurement would enable determination of the integrity of the secondary reflector to determine how much noise temperature is coming from the ground (see Eq. (3)).

\section{B. Problem Areas}

An important part of the development of dielectrically loaded dichroic plates is the accurate determination of the properties of the dielectric material to be used for the plates. A dichroic plate with over 1000 holes and loaded with dielectric material is expensive to fabricate. If the dielectric constant is not closely known, then it may not be possible to fine-tune the plate by trimming the plate thickness. The dielectric constant needs to be determined for the density state that the plugs will be in after installation by whatever method is employed for locking the plugs in place. Accurate determination of the dielectric constant is a time-consuming task that is not generally appreciated or understood as being a necessary part of this developmental work.
Another technical challenge related to dichroic plate design lies in finding a suitable material that has low loss, but at the same time is mechanically durable, chemically inert, and commercially available at reasonable cost. The selection and evaluation of materials presents the same type of technical challenge that developers of radomes have had to face in previous and current decades.

Another technical problem that currently needs to be solved is the development of an improved computer program that accurately predicts the passbands for the dielectrically loaded plate. Currently, the Chen-Otoshi program version being used is a 10-waveguide mode program. Revising the program to include twice as many modes might result in a computer program tool that would be invaluable for designing dichroic plates. Also, the incorporation of a version that provides for arbitrary aperture shapes other than round holes might prove invaluable.

Another unsolved technical problem at this point of development is that of knowing the CW power at which arcing across the holes will occur. Some preliminary calculations show that breakdown will not occur at $100 \mathrm{~kW}$ of $\mathrm{CW}$ power, but experimental verifications need to be made.

\section{Summary and Conclusions}

The primary objectives of this study have been accomplished. It has been demonstrated that a dichroic plate having two passbands at the desired frequencies can be developed. Over the frequency range of 7.0 to $9.0 \mathrm{GHz}$, good agreement was obtained between theory and experiment for perpendicular polarization. For parallel polarization, the measured passband occurred at a center frequency that was about 55 $\mathrm{MHz}$ higher than theoretical predictions for both uplink and downlink passbands. The noise temperature of the Teflonloaded plate is determined to be about $3.0 \mathrm{~K}$ if corrections are made for about $1.5 \mathrm{~K}$ error that is attributable to measurement setup problems. This $3.0 \mathrm{~K}$ noise temperature value includes a contribution of about $1.3 \mathrm{~K}$ due to dissipative losses of the Teflon plugs. Antenna pattern measurements showed that for the dielectrically loaded plate at 30-deg incidence, there was only one reflection lobe, as theoretically predicted, and that this lobe had a magnitude that correlated with the data obtained from insertion loss measurements.

Immediate technical challenges and tasks that remain for developing a dielectrically loaded dichroic plate for the DSN are (1) the development of a Pyle waveguide type dielectrically filled dichroic plate; (2) improvement in the computer program to theoretically design the plates; and (3) experimental 
verification that arcing does not occur for a dielectrically loaded plate at high $\mathrm{CW}$ power levels at about $100 \mathrm{~kW}$.

Long-term projects include (1) research into lower loss type dielectric materials; (2) research into different types of apertures (other than round holes and Pyle waveguide) for develop- ing plates that operate over a broader bandwidth and over a greater range of incidence angles; (3) development of cornputer programs and theory to design new classes of improved dichroic plates; and (4) development of less expensive, less time-consuming techniques for experimental verifications of performance.

\section{Acknowledgments}

R. Clauss, of the TDA Technology Development Office, provided the initial idea of designing a dielectrically loaded plate for dual passband purposes. P. Parsons, of the TDA Engineering Office, provided technical requirements for the dichroic plate project.

The accomplishments of this project were made possible because of a cooperative team effort by various individuals of the Radio Frequency and Microwave Subsystems Section. Management support and encouragement came from Section Manager W. Imbriale, RTOP Manager A. Cha, and Group Supervisor D. Bathker. Technical and manpower assistance were provided by the Microwave Engineering Group on Automatic Network Analyzer measurements on Teflon dielectric samples, the Microwave Electronics Group on insertion loss and noise temperature measurements, and the Antenna and Microwave Development Group on all phases of the experimental work. Appreciation is expressed to J. Withington, of the Antenna and Microwave Development Group, who allowed temporary rearrangement of his work in order to permit antenna patterns to be taken of the dichroic plates in the 60 -ft anechoic chamber. Invaluable computer-programming and datareduction assistance was provided by $\mathrm{E}$. Andres, JPL contractor. Appreciation is expressed to many unnamed technical and support people who directly or indirectly contributed to this project. 


\section{References}

[1] D. A. Bathker, "Dual Frequency Dichroic Feed Performance," Antennas for Avionics, AGARD-CP-139, 26th Meeting of Avionics Panel, pp. 29-1-29-10, June 1974.

[2] C. C. Chen, "Diffraction of Electromagnetic Waves by a Conducting Screen Perforated Periodically with Circular Holes," IEEE Trans. Microwave Theory and Techniques, vol. MTT-19, pp. 475-481, May 1971.

[3] C. C. Chen, "Transmission of Microwave Through Perforated Flat Plates of Finite Thickness," IEEE Trans. Microwave Theory and Techniques, vol. MTT-21, pp. 1-6, January 1973.

[4] P. D. Potter, Improved Dichroic Reflector Design for the 64-Meter Antenna S- and $X$-Band Feed Systems, JPL Technical Report 32-1526, vol. XIX, Jet Propulsion Laboratory, Pasadena, California, pp. 55-62, February 15, 1974.

[5] Reference Data for Radio Engineers, Fifth Edition, New York: International Telephone and Telegraph, pp. 4-28-4-29, 1968.

[6] W. B. Weir, "Automatic Measurement of Complex Dielectric Constant and Permeability at Microwave Frequencies," Proc. IEEE, pp. 33-36, January 1974.

[7] Hewlett-Packard, "Measuring the Dielectric Constant of Solids with the HP 8510 Network Analyzer," Hewlett-Packard Product Note 8510-3, August 1, 1985.

[8] A. C. Ludwig, "The Definition of Cross Polarization," IEEE Trans. Antenna Propagat., vol. AP-21, pp. 116-119, January 1973. 
Table 1. Design data" and theoretical passband insertion losses of dielectric filled hole plate (Plate \#1) for phi = $90 \mathrm{deg}$ and incidence angle $=\mathbf{3 0} \mathrm{deg}$

\begin{tabular}{llcccl}
\hline Polarization & Band & \multicolumn{2}{c}{ Passband insertion loss, dB } & Comments \\
\cline { 3 - 5 } & & $\begin{array}{c}\text { Start } \\
\text { frequency }\end{array}$ & $\begin{array}{c}\text { Mid } \\
\text { frequency }\end{array}$ & $\begin{array}{c}\text { End } \\
\text { frequency }\end{array}$ & \\
\hline Perpendicular & Uplink & 0.02 & 0.06 & 0.14 & $\begin{array}{l}\text { Not too good at mid- } \\
\text { band and end of band }\end{array}$ \\
Perpendicular & Downlink & 0.01 & 0.02 & 0.06 & $\begin{array}{l}\text { Not too good at end } \\
\text { of band }\end{array}$ \\
Parallel & Uplink & 0.13 & 0.06 & 0.02 & $\begin{array}{l}\text { Not too good at start } \\
\text { and mid-band }\end{array}$ \\
Parallel & Downlink & 0.04 & 0.02 & 0.01 & \\
\hline
\end{tabular}

${ }^{\mathrm{a}}$ Hole diameter $=0.803$ in., hole-to-hole spacing $=0.850$ in., plate thickness $=1.580$ in.

Hole medium dielectric constant $=2.04$.

Loss tangent $=0.00015$.

${ }^{\text {Uplink, downlink }}=7170 \pm 30 \mathrm{MHz}, 8430 \pm 30 \mathrm{MHz}$.

Notes:

(1) The insertion loss values include dissipative loss due to the loss tangent of Teflon.

(2) At some of the passband frequencies, the results are not good at 15- or 45-deg incidence angles. 
Table 2. Design data" and theoretical passband insertion losses of DSN air-filled hole plate (Plate \#4) for phi = $90 \mathrm{deg}$ and incidence angle $=\mathbf{3 0} \mathrm{deg}$

\begin{tabular}{lcccc}
\hline \multirow{2}{*}{ Polarization } & Band $^{\mathrm{b}}$ & \multicolumn{3}{c}{ Passband insertion loss, $\mathrm{dB}$} \\
\cline { 3 - 5 } & & $\begin{array}{c}\text { Start } \\
\text { frequency }\end{array}$ & $\begin{array}{c}\text { Mid } \\
\text { frequency }\end{array}$ & $\begin{array}{c}\text { End } \\
\text { frequency }\end{array}$ \\
Perpendicular & Downlink & 0.000 & 0.009 & 0.037 \\
Parallel & Downlink & 0.025 & 0.004 & 0.001 \\
\hline
\end{tabular}

aPyle waveguide dimensions $=0.866$ and 0.900 in., respectively, for perpendicular and parallel polarizations. The corresponding cutoff wavelengths of the Pyle waveguide are 1.511 and 1.539 in. (see note 1 below for equivalent round-hole dimensions).

Hole-to-hole spacing $=0.940$ in., plate thickness $=1.408$ in .

Hole medium dielectric constant $=1.00$.

Loss tangent $=0.00000$.

${ }^{b}$ Downlink $=8415 \pm 30 \mathrm{MHz}$.

Notes:

(1) This DSN Plate has a Pyle waveguide aperture (see [4]) rather than a round-hole aperture. The equivalent round-hole diameters corresponding to the 0.866- and 0.900-inch Pyle waveguide dimension are respectively 0.886 and 0.902 inches. These round-hole dimensions were used to compute the theoretical insertion losses.

(2) This plate has no dielectric dissipative loss term associated with insertion loss.

(3) At some of the passband frequencies, the results were not too good at 15- or 45-deg incidence angles.
Table 3. Noise temperature test results for dichroic plates at phi $=\mathbf{9 0}$ deg and theta $=\mathbf{3 0} \mathrm{deg}$

\begin{tabular}{|c|c|c|c|c|c|c|}
\hline \multirow{2}{*}{ Description } & \multicolumn{3}{|c|}{$\begin{array}{c}\text { Perpendicular } \\
\text { polarization } \\
\text { noise temperature, } \mathrm{K}\end{array}$} & \multicolumn{3}{|c|}{$\begin{array}{c}\text { Parallel } \\
\text { polarization } \\
\text { noise temperature, } \mathrm{K}\end{array}$} \\
\hline & 8400 & 8450 & 8500 & 8400 & 8450 & 8500 \\
\hline $\begin{array}{l}\text { Plate \#1 } \\
\text { with Teflon } \\
\text { plugs }\end{array}$ & 4.7 & 4.3 & 4.0 & 11.0 & 5.2 & 4.0 \\
\hline $\begin{array}{l}\text { Plate \#4 } \\
\text { air dielectric }\end{array}$ & 1.5 & 3.2 & 1.6 & 1.6 & 1.6 & 1.7 \\
\hline $\begin{array}{l}\text { Difference } \\
\text { in noise } \\
\text { temperature }\end{array}$ & 3.2 & 1.1 & 2.4 & 9.4 & 3.6 & 2.3 \\
\hline \multicolumn{7}{|l|}{ Notes: } \\
\hline (1) & $\begin{array}{l}\text { red mic } \\
\text { for per } \\
\text { arizati }\end{array}$ & $\begin{array}{l}\text { requen } \\
\text { ndicul } \\
\text { for } \mathrm{Pla}\end{array}$ & $\begin{array}{l}\text { of the } \mathrm{i} \\
\text { polariza } \\
\# 1\end{array}$ & $\begin{array}{l}\text { sertion } \\
\text { on and }\end{array}$ & $\begin{array}{l}\text { ss passt } \\
500 \mathrm{MI}\end{array}$ & $\begin{array}{l}\text { ids are } \\
\text { for }\end{array}$ \\
\hline $\begin{array}{l}\text { (2) The me } \\
8415 \mathrm{M} \\
\text { Plate \#4 }\end{array}$ & $\begin{array}{l}\text { red mis } \\
\text { for bo }\end{array}$ & $\begin{array}{l}\text { requen } \\
\text { perpen }\end{array}$ & $\begin{array}{l}\text { of the } \\
\text { cular an }\end{array}$ & $\begin{array}{l}\text { sertion } \\
\text { paralle }\end{array}$ & $\begin{array}{l}\text { ss pass } \\
\text { olariza }\end{array}$ & $\begin{array}{l}\text { ads is } \\
\text { ns for }\end{array}$ \\
\hline
\end{tabular}

Table 4. Correlation of the relative level of the reflection lobe to measured insertion loss for Plate \#1

\begin{tabular}{lccclll}
\hline & & Relative & Equivalent & \multicolumn{2}{c}{ Measured } \\
Pattern & $\begin{array}{c}\text { Frequency, } \\
\mathrm{MHz}\end{array}$ & $\begin{array}{c}\text { level of } \\
\text { reflection } \\
\text { lobes, dB }\end{array}$ & $\begin{array}{c}\text { insertion } \\
\text { loss, dB }\end{array}$ & & \multicolumn{2}{c}{ insertion loss } \\
\hline E-Plane & 7168 & -16.0 & 0.11 & Parallel & 0.2 \\
H-Plane & 7168 & -21.0 & 0.03 & Perpendicular & 0.2 \\
E-Plane & 8425 & -20.0 & 0.04 & Parallel & 0.2 \\
H-Plane & 8425 & -21.0 & 0.03 & Perpendicular & 0.05 \\
\hline
\end{tabular}

Notes:

(1) Data for reflection lobe levels were obtained from patterns of Plate \#1 and taken relative to the peak of the corresponding horn-only pattern.

(2) Data for the measured insertion loss were obtained from Fig. 10 and 11 , respectively, for perpendicular and parallel polarizations. 
ORICINAL PAGE IS

OF POOR QUALITY

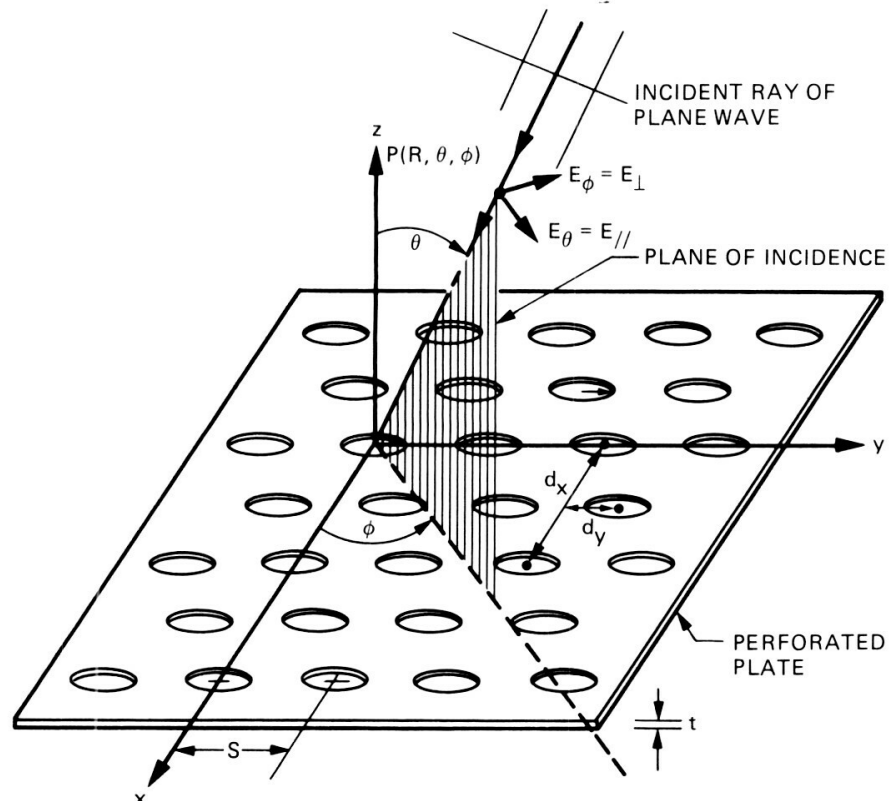

Fig. 1. Dichroic plate geometry

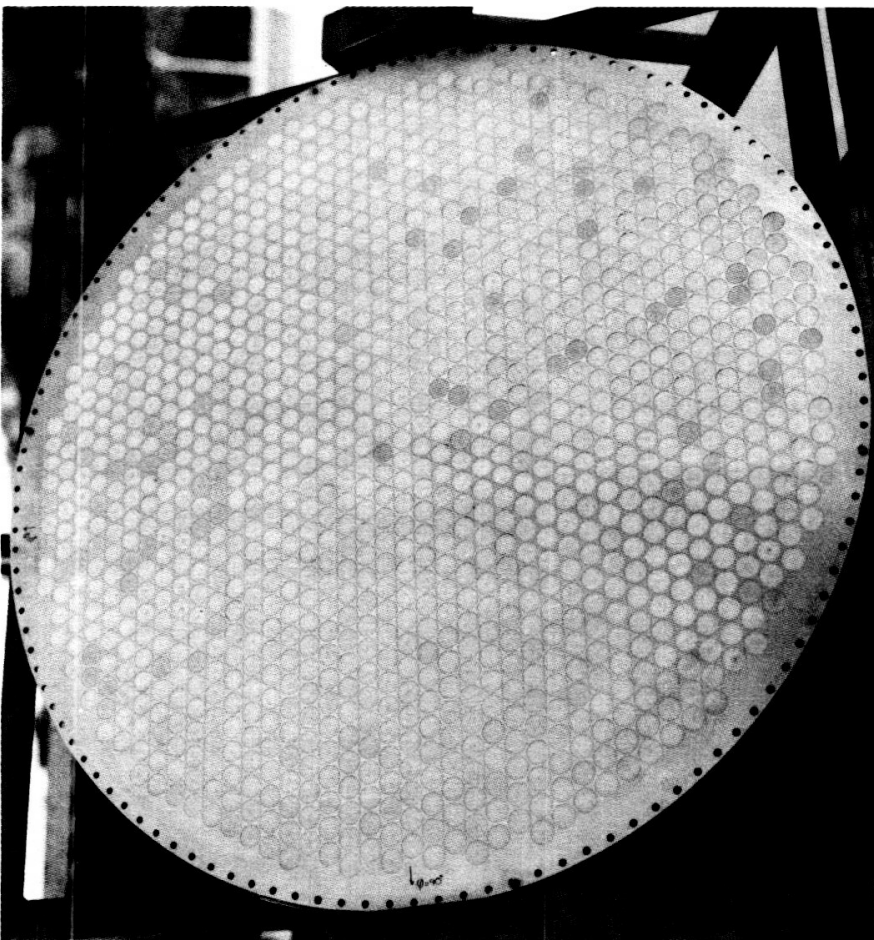

Fig. 2. Now X-band dual passband dichroic plate with holes filled with Tefion plugs; this plate is referred to as Plate \#1 in the toxt

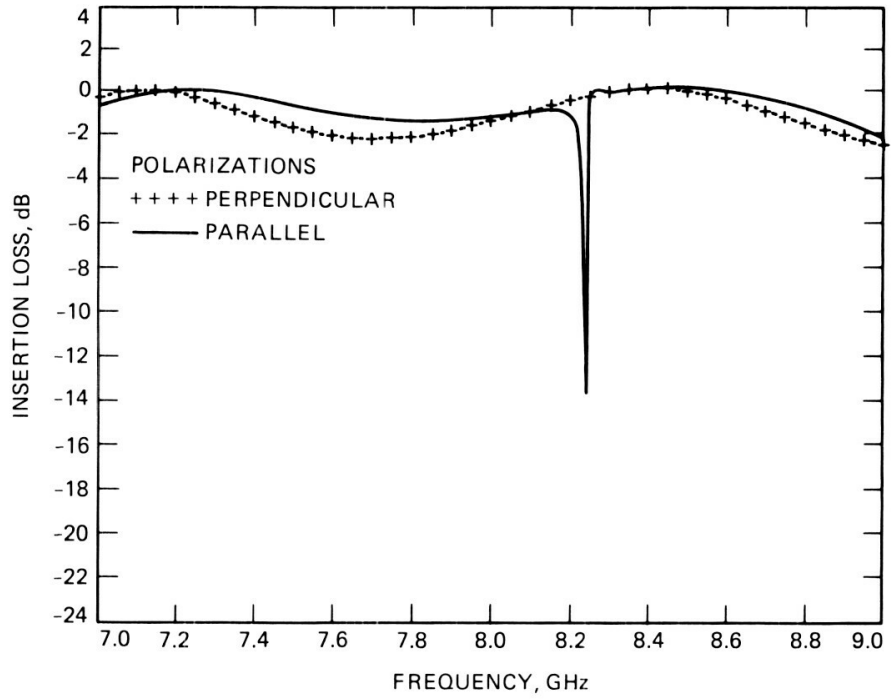

Fig. 3. Theoretical insertion loss versus frequency for the dielectrically loaded plate (Plate \#1) for perpendicular and parallel polarizations

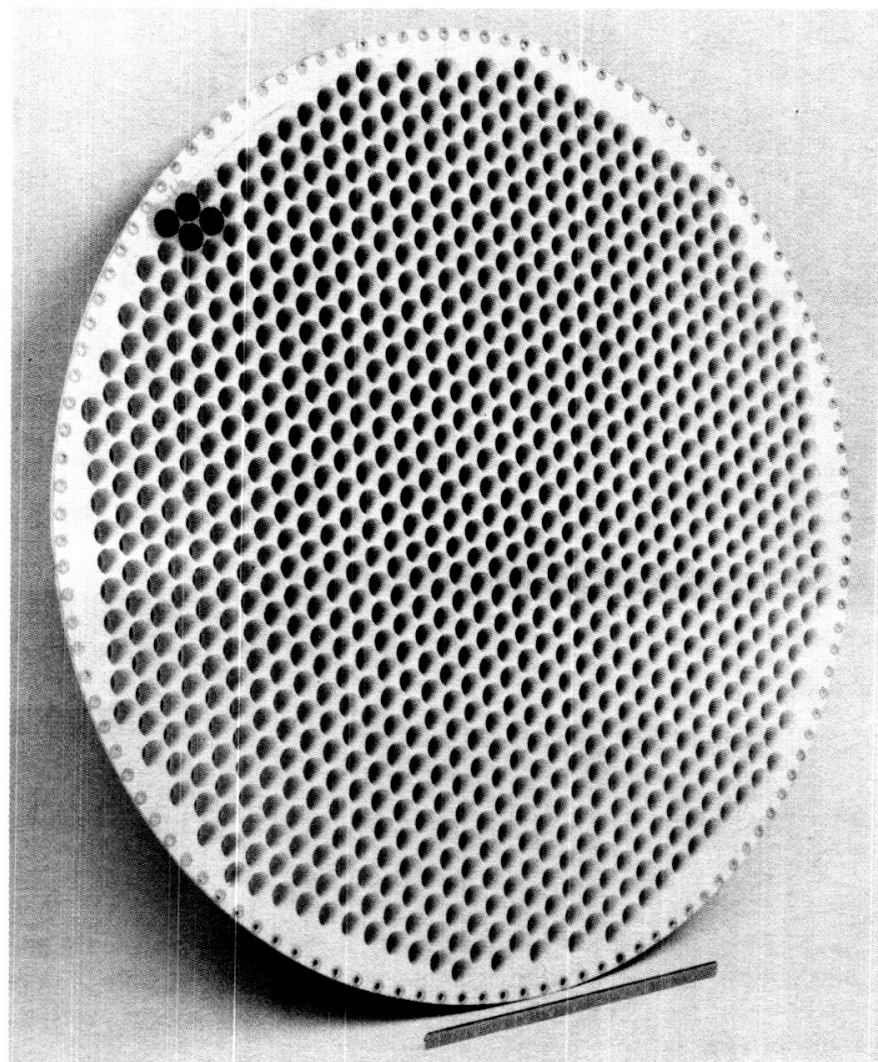

Fig. 4. Prototype DSN X-band dichrolc plate; this plate is referred to as Plate \#4 in the toxt 


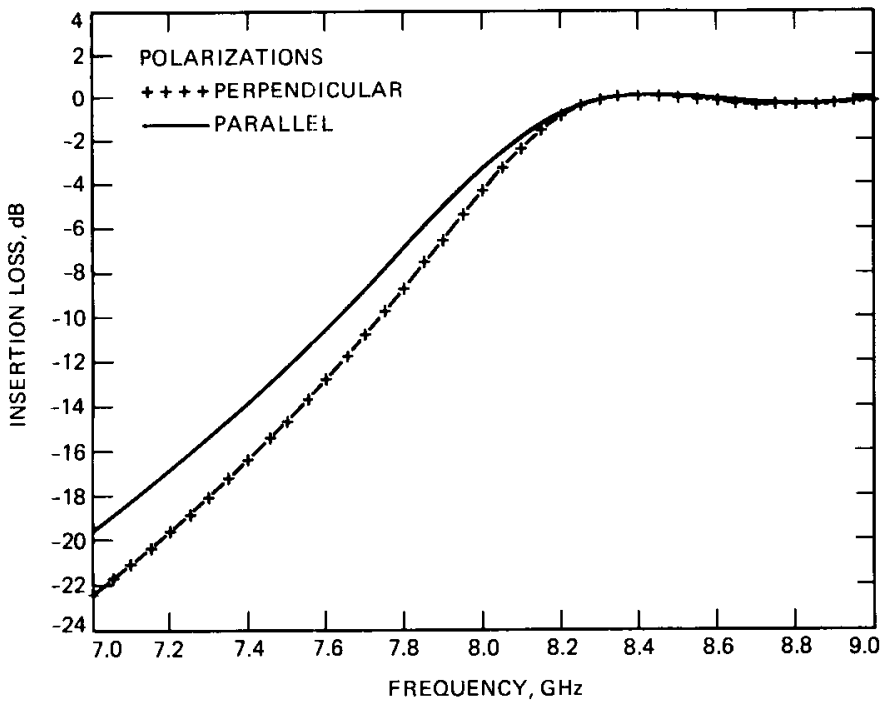

Fig. 5. Theoretical insertion loss versus frequency for the DSN plate (Plate \#4) for perpendicular and parallel polarizations

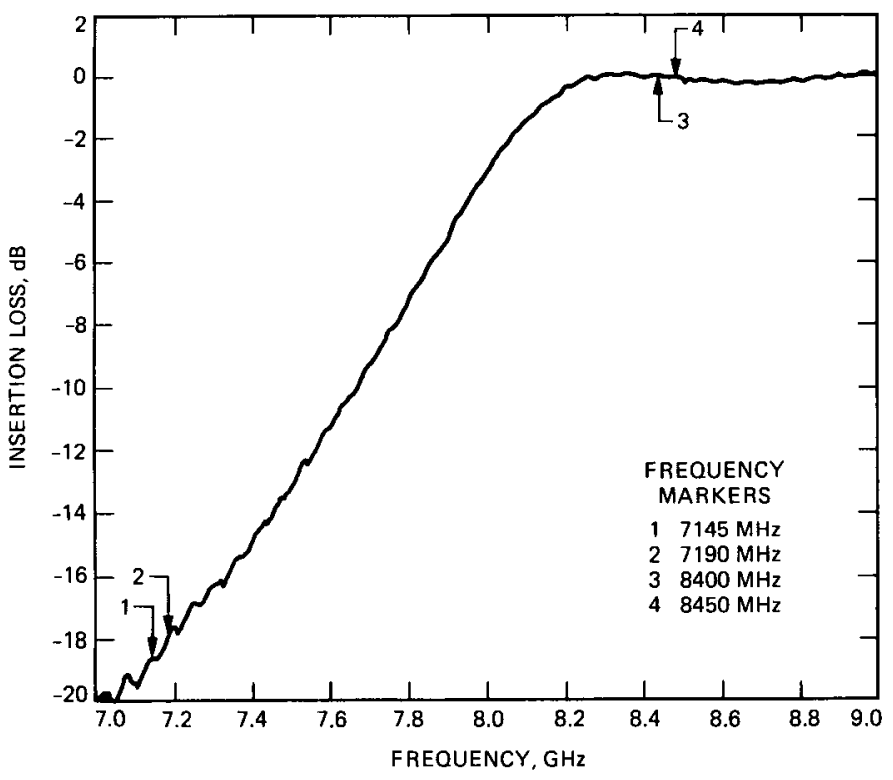

Fig. 6. Measured insertion losses of Plate \#4 for perpendicular polarization, 30-deg incidence angle, and phi $=90$-deg hole pattern orientation; measurements were made in the 60-ft anechoic chamber test setup

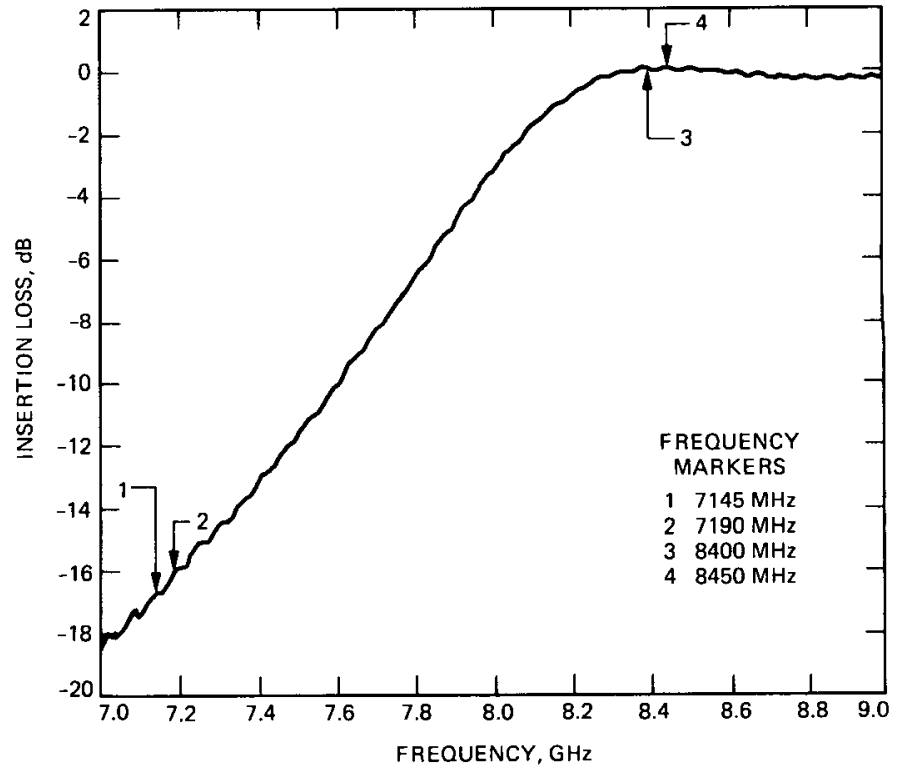

Fig. 7. Measured insertion losses of Plate \#4 for parallel polarization, 30-deg incidence angle, and phi $=90$-deg hole pattern orientation; measurements were made in the 60-ft anechoic chamber setup 


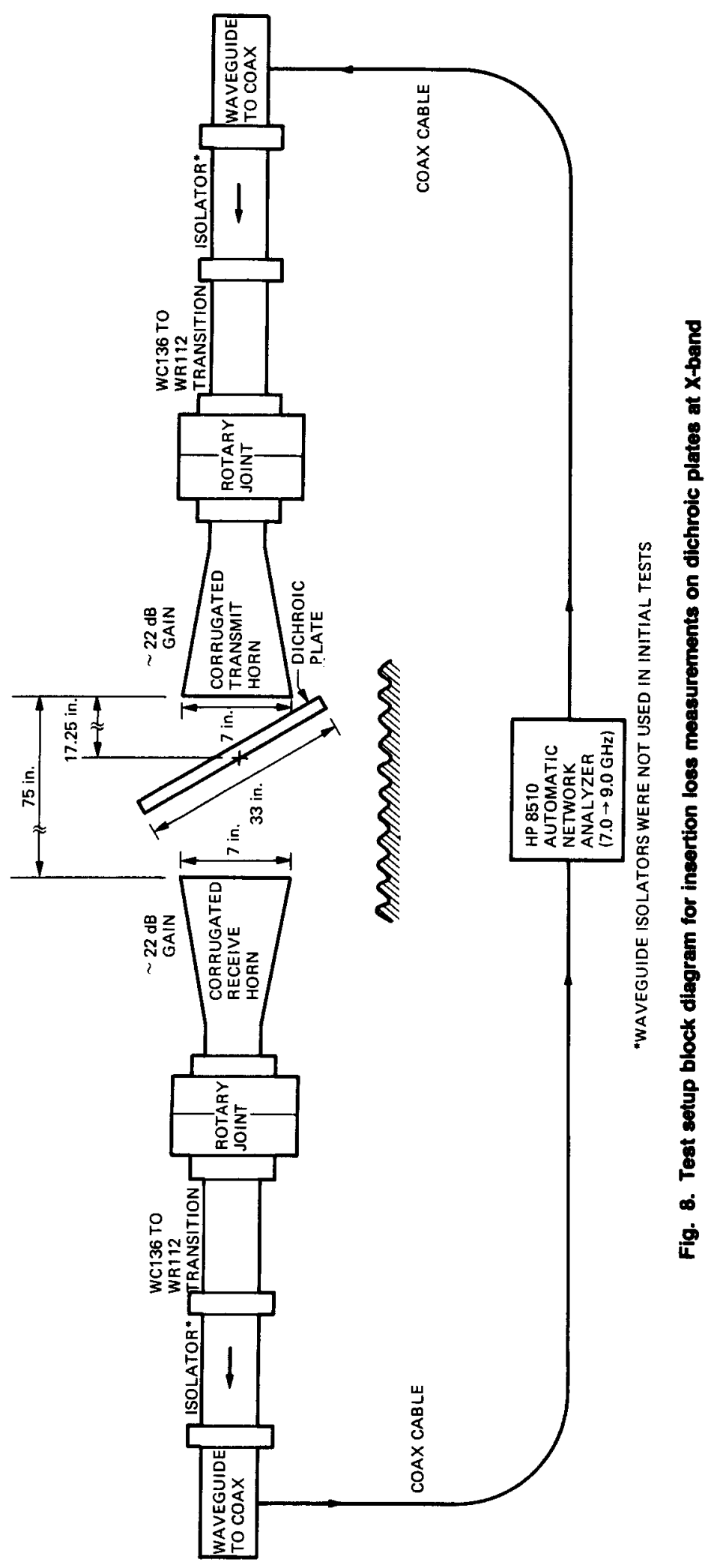




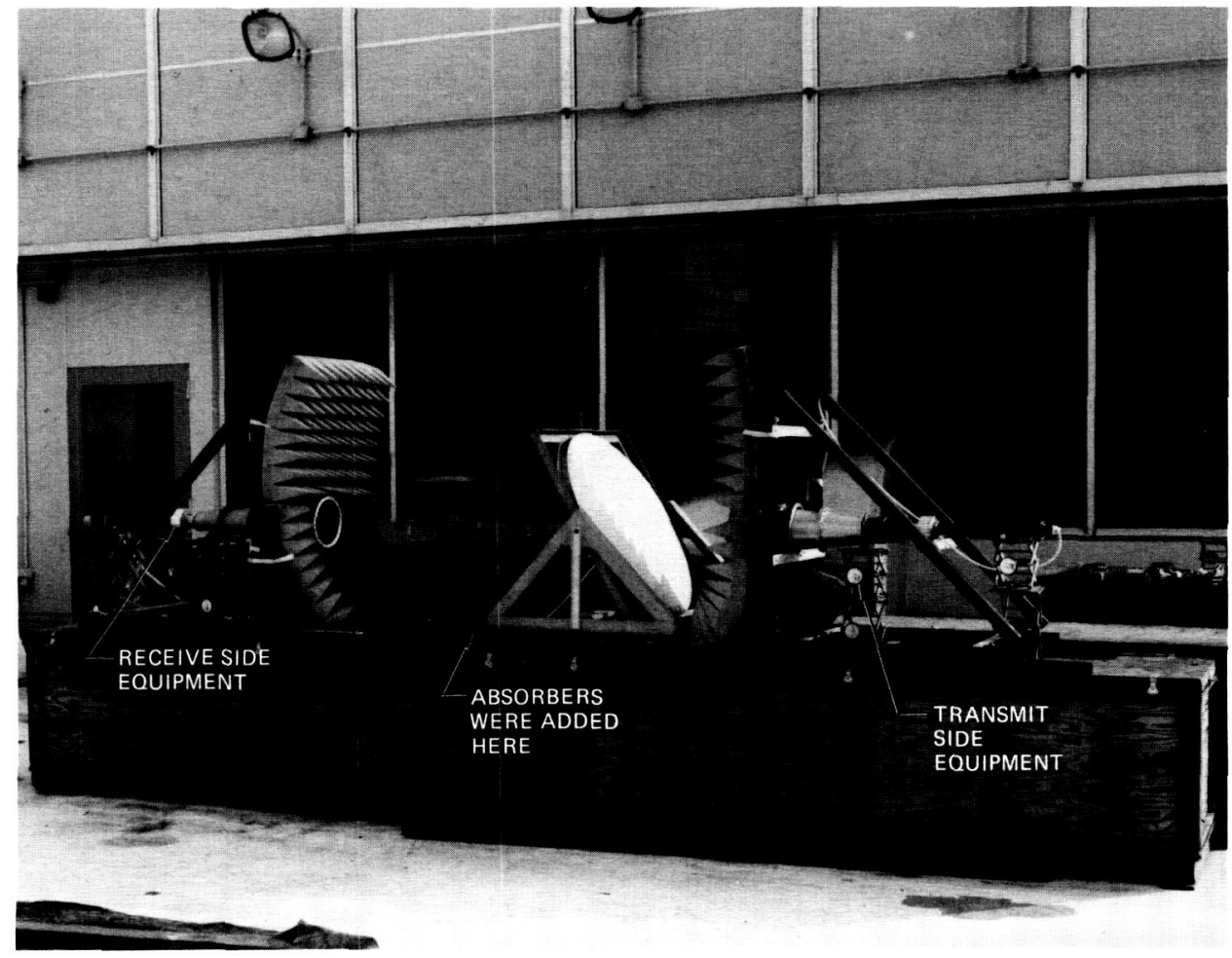

Fig. 9. Outdoor X-band test setup (roof, Building 238, JPL) for insertion loss measurements on dichroic plates; absorbers (not shown in the picture) were placed at the indicated area and on plate test fixture in order to minimize multipath signals reflecting into the receive horn

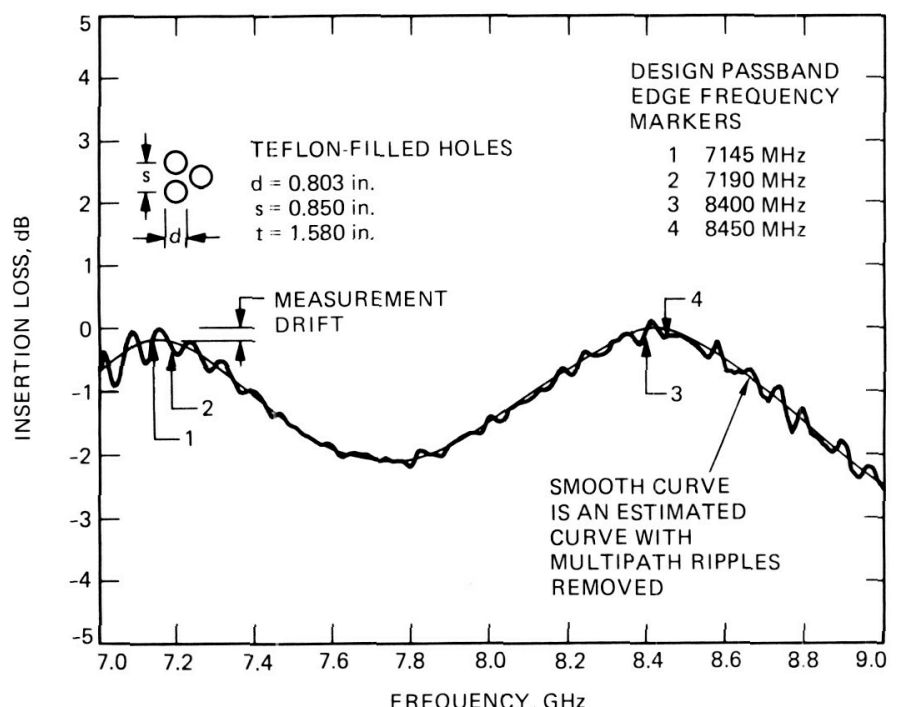

Fig. 10. Measured insertion loss of Plate \#1 for perpendicular polarization, 30 -deg incidence angle, and $\mathrm{phi}=90$-deg hole pattern orientation; measurements were made in the outdoor test setup (see Fig. 9)

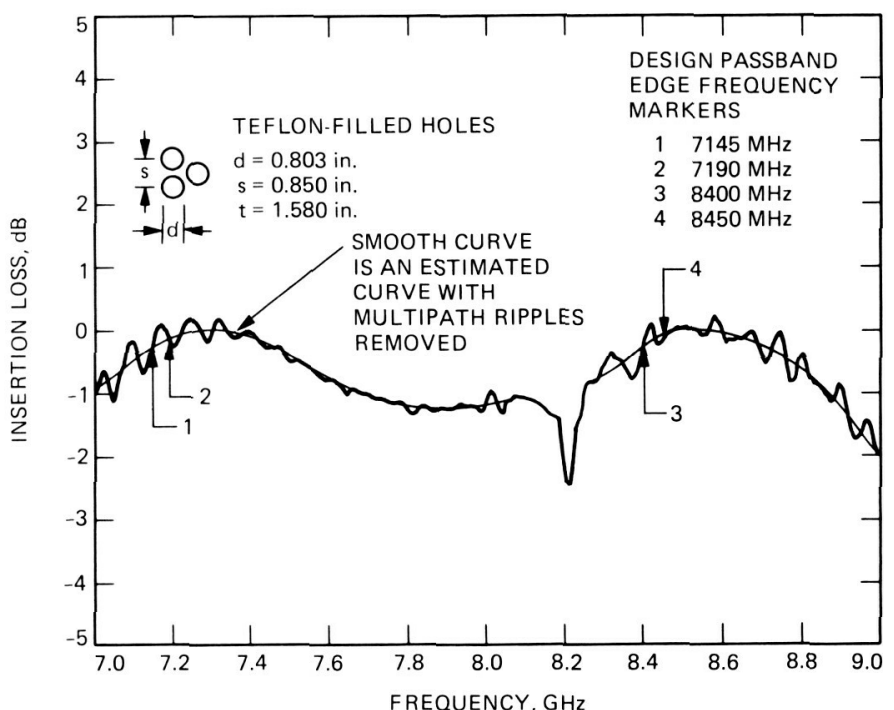

Fig. 11. Measured insertion losses of Plate \#1 for parallel polarization, 30-deg incidence angle, and phi $=90$-deg hole pattern orientetion; measurements were made in the outdoor test setup 


\section{ORIGINAL PAGE IS \\ OF POOR QUALITY}

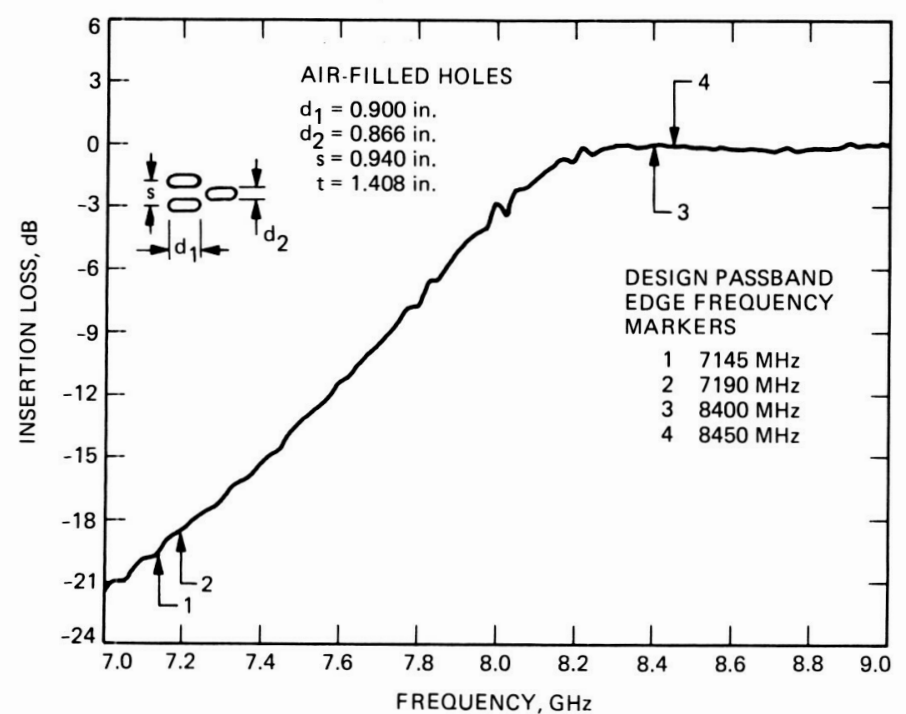

Fig. 12. Measured insertion losses of Plate \#4 for perpendicular polarization, $30-\mathrm{deg}$ incidence angle, and $\mathrm{phi}=90$-deg hole pattern orientation; measurements were made in the outdoor test setup

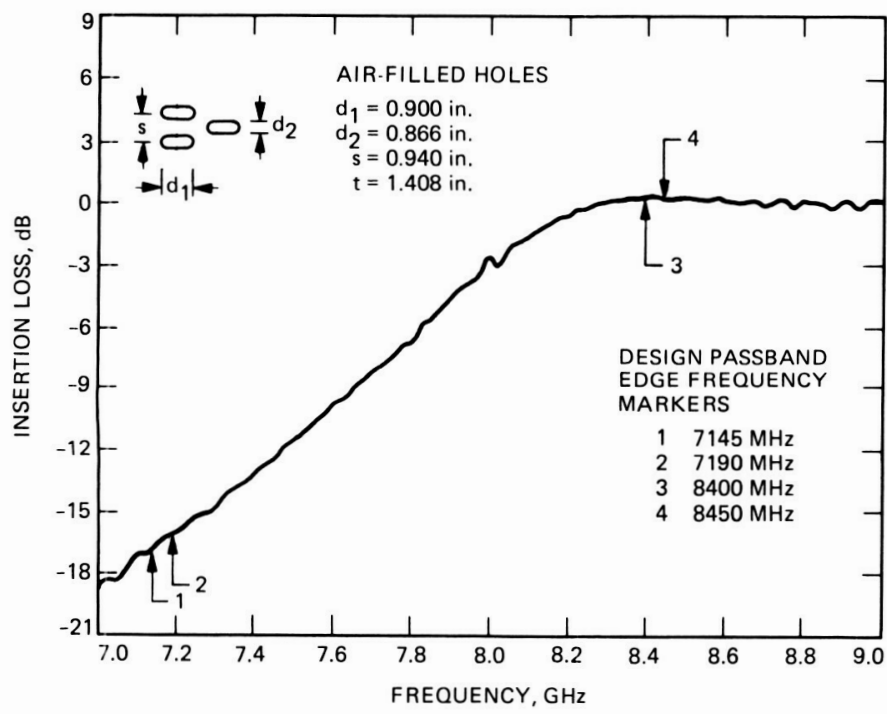

Fig. 13. Measured insertion losses of Plate \#4 for parallel polarization, 30-deg incidence angle, and phi $=90$-deg hole pattern orientation; measurements were made in the outdoor test setup

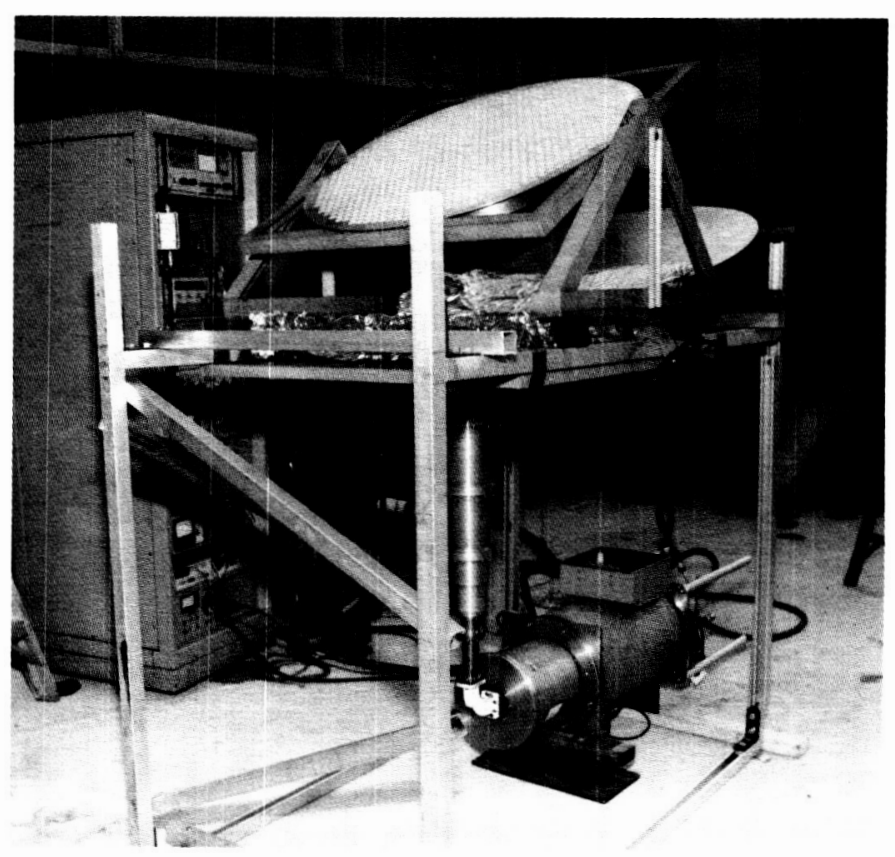

Fig. 14. Test setup for noise temperature measurements of Plate \#1 for perpendicular polarization, 30-deg incidence angle, and phi $=90$-deg hole pattern orientation (see Fig. 1)

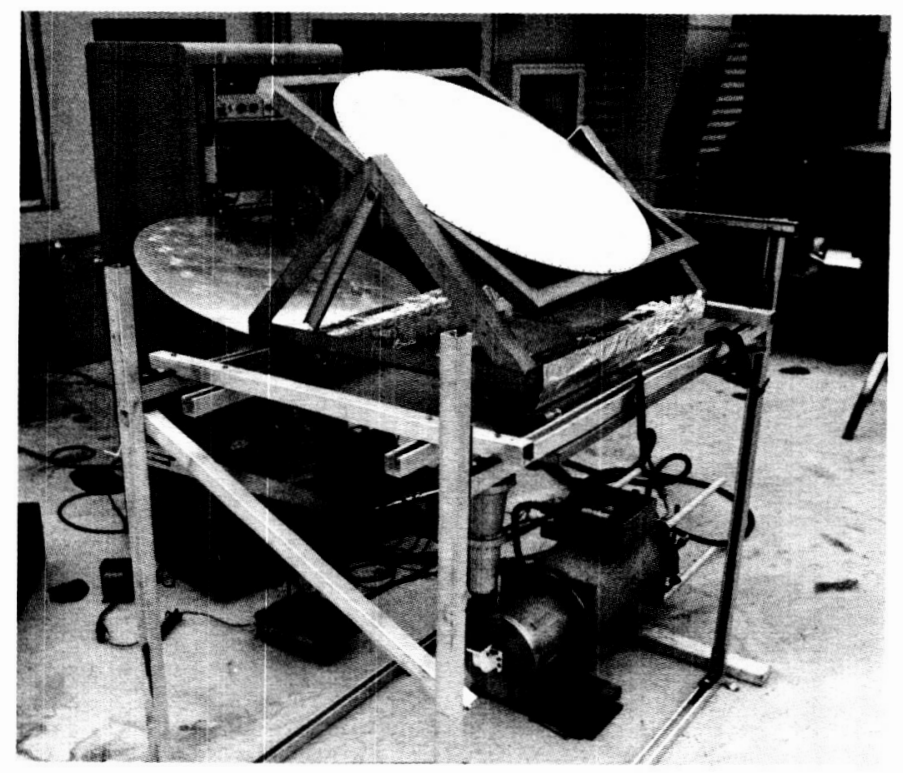

Fig. 15. Test setup for noise temperature measurements of Plate \#1 for parallel polarization, 30-deg incidence angle, and phi = 90-deg hole pattern orientation (see Fig. 1) 


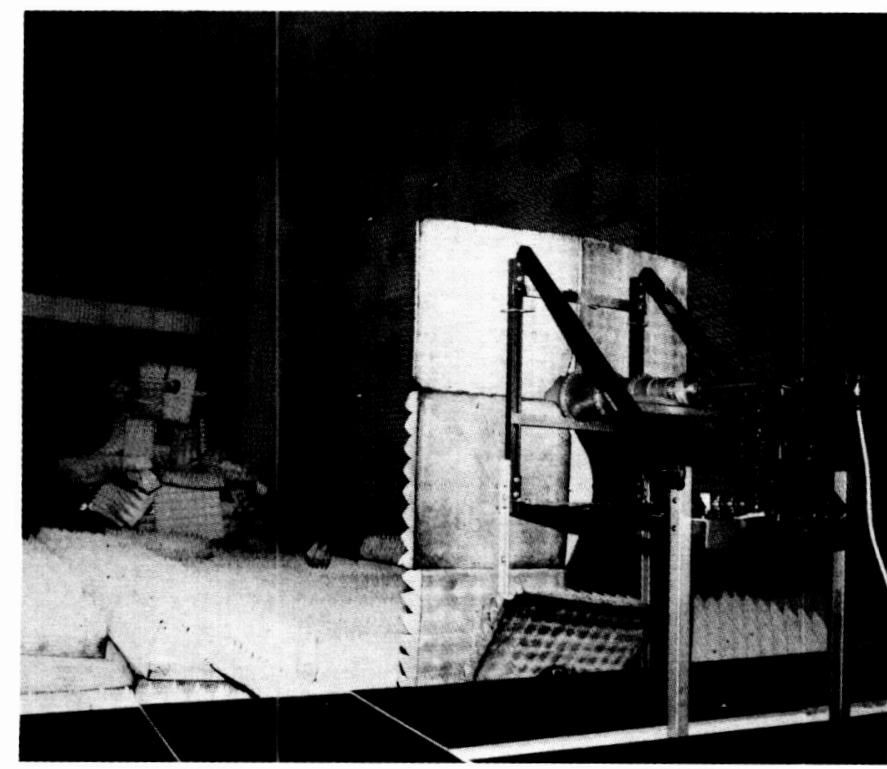

Fig. 16. Anechoic chamber antenna pattern measurement test setup for measurement of X-band horn only with axis of rotation aligned with the horn phase center

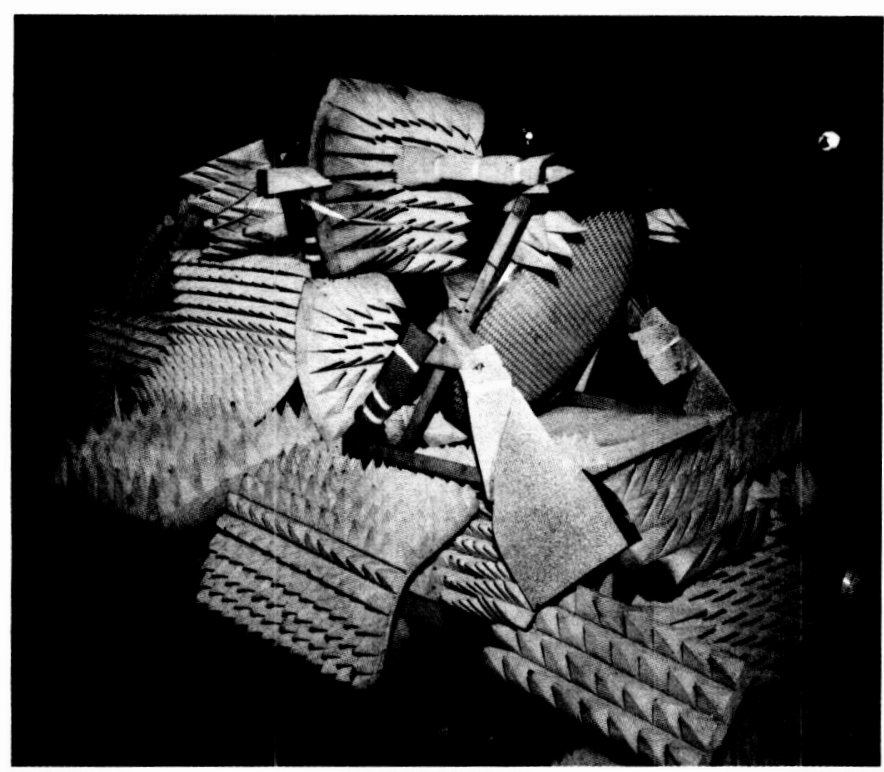

Fig. 17. Anechoic chamber antenna pattern measurement test setup for measurement of X-band horn and dichroic plate at 30-deg incidence angle with plate holder upright; in this configuration, the main reflection lobe is absorbed by the ceiling of the anechoic chamber, but scattering from plate in the orthogonal plane is recorded

\section{ORIGINRL PAEE IS OF POOR QUALTY}

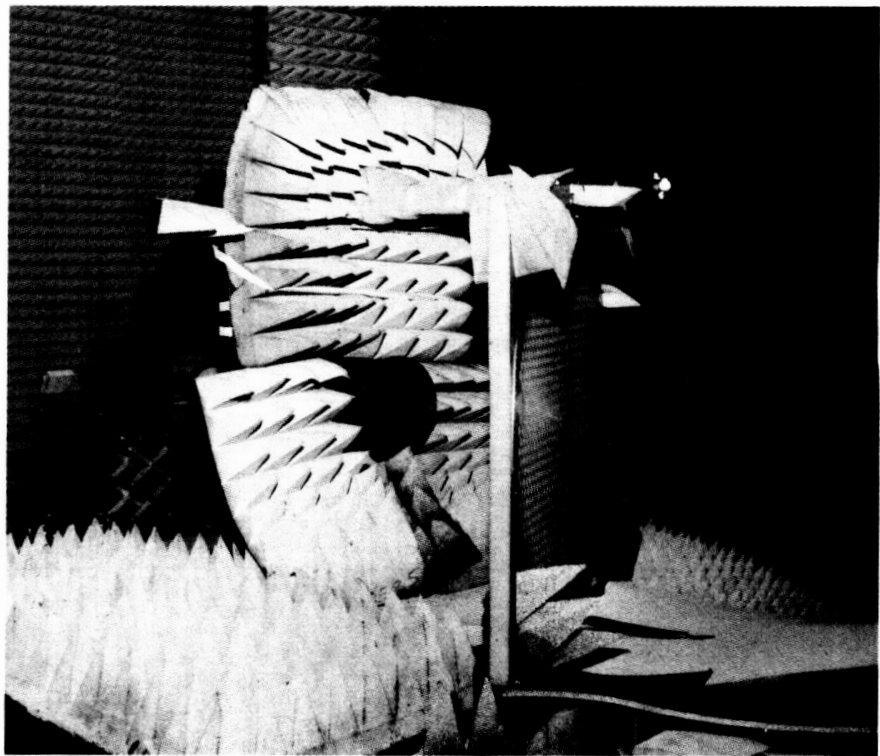

Fig. 18. Anechoic chamber antenna pattern measurement test setup for measurement of X-band horn and dichroic plate at 30-deg incidence angle with plate holder flopped $90 \mathrm{deg}$ on its side; this configuration enables recording the main reflection lobe scattered from the dichroic plate 


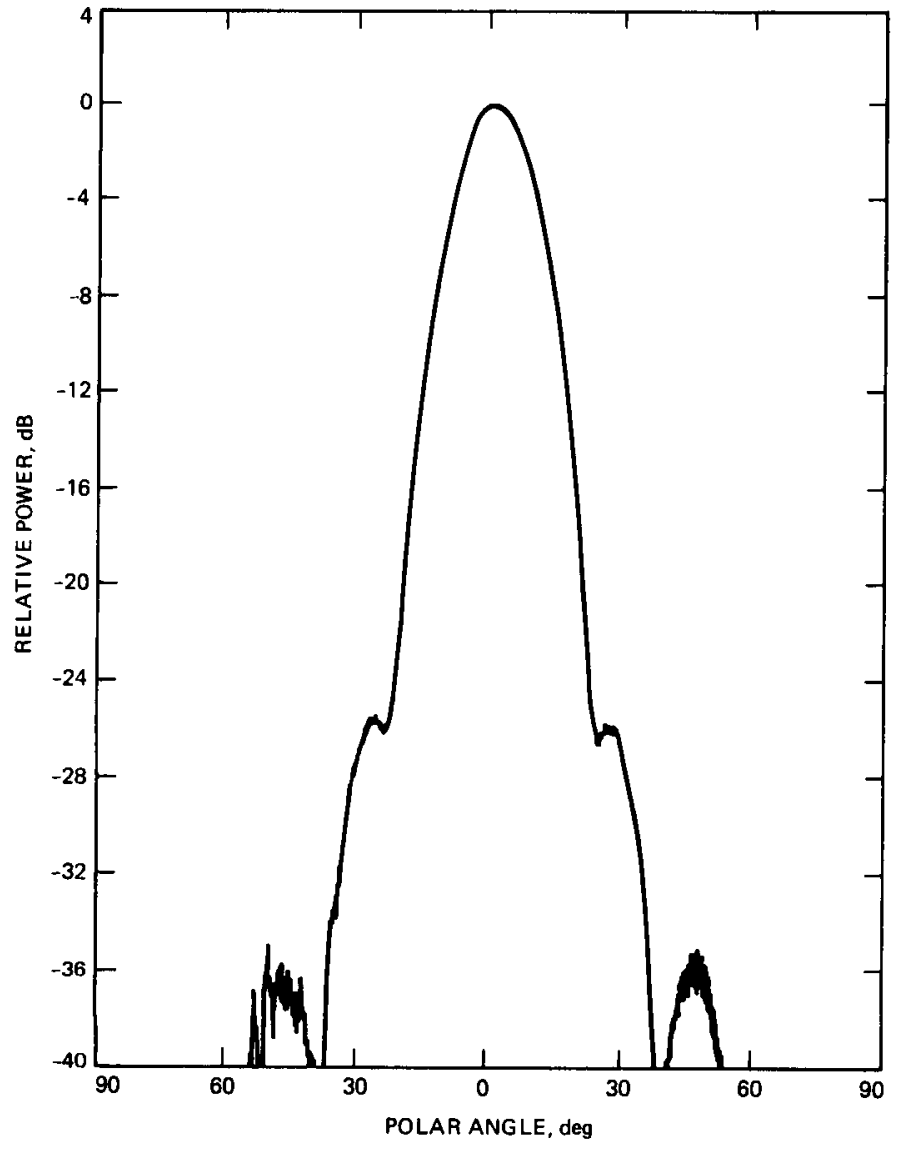

Fig. 19. E-plane pattern for X-band horn only at $7168 \mathrm{MHz}$

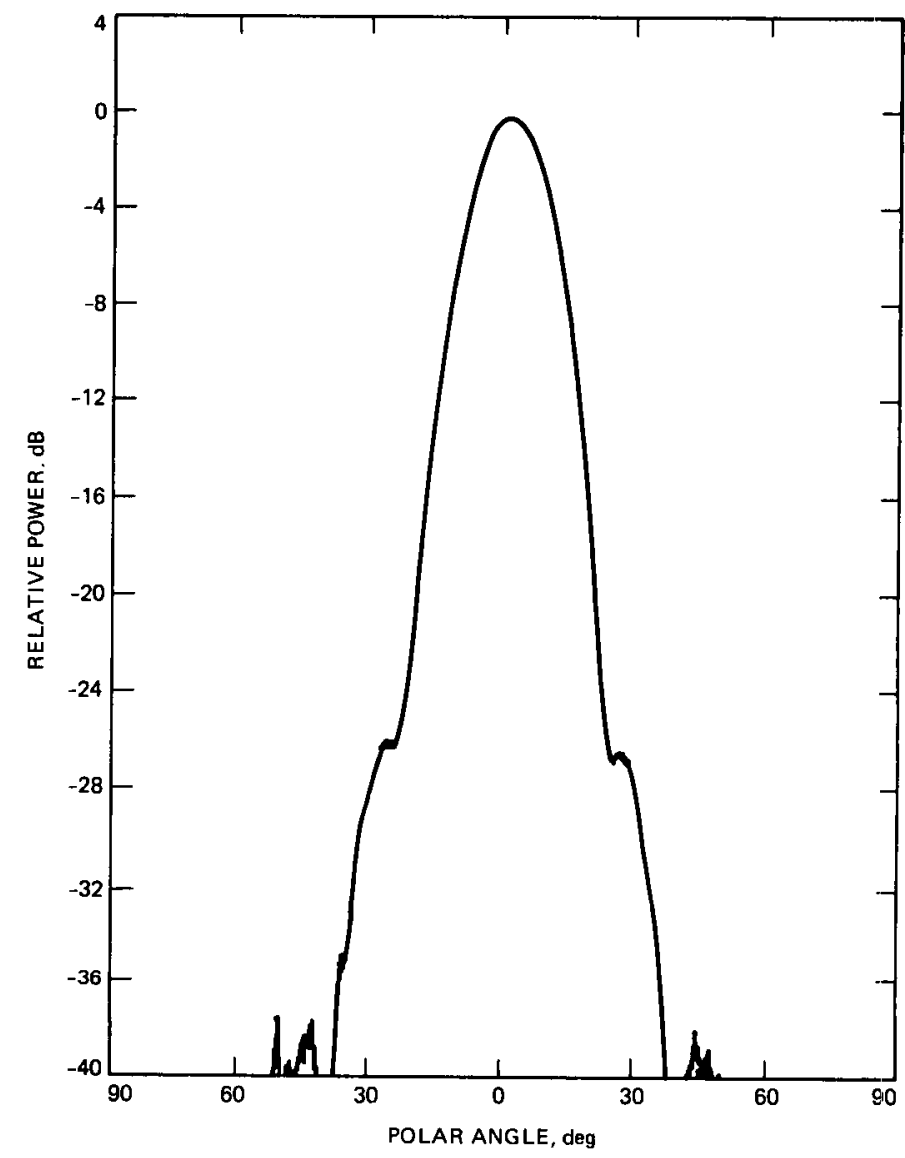

Fig. 20. H-piane pattern for X-band horn only at $7168 \mathrm{MHz}$ 


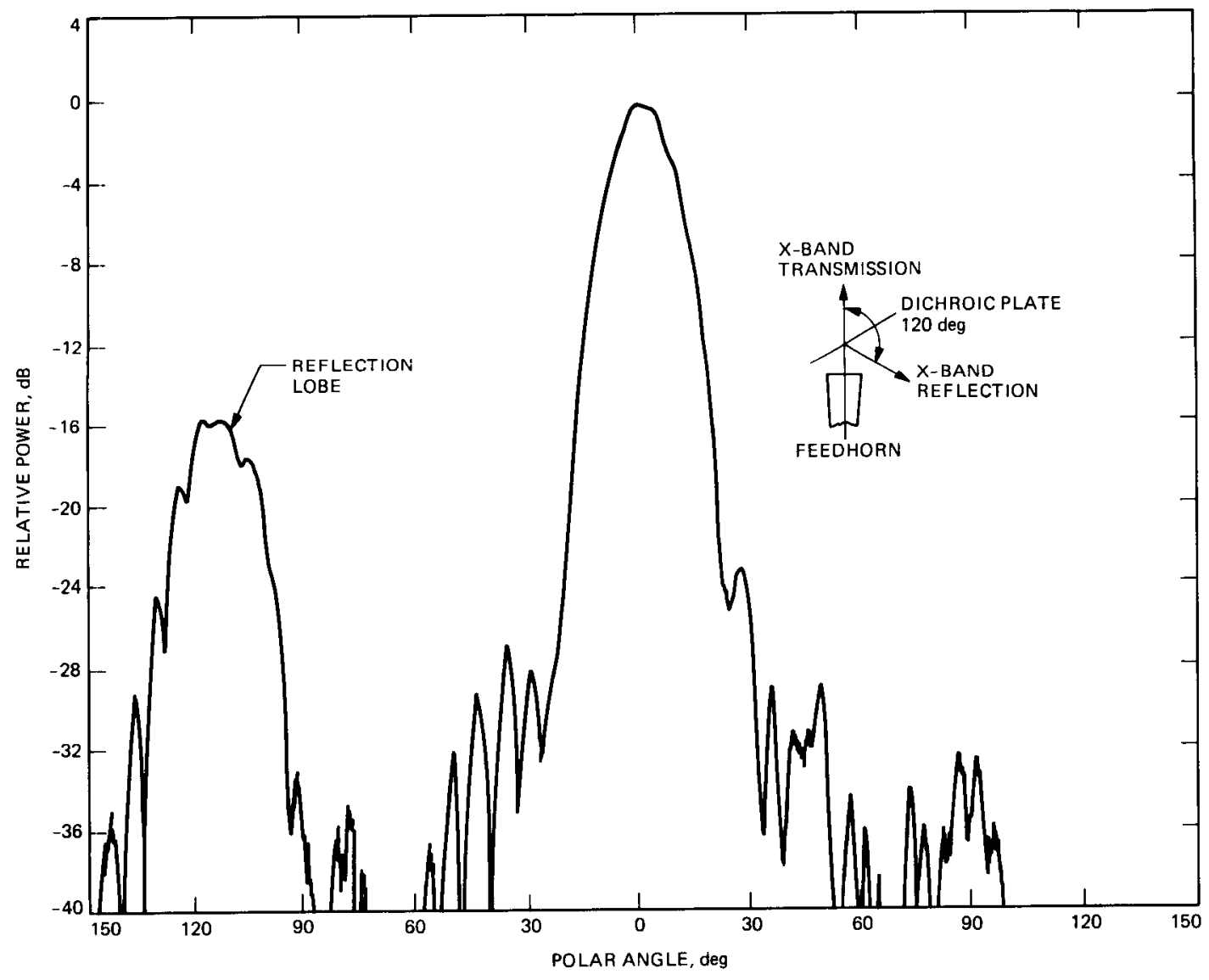

Fig. 21. E-plane pattern for X-band horn plus dichroic Plate \#1 at $7168 \mathrm{MHz}$ with plate holder flopped $90 \mathrm{deg}$ on its side; plate is in the theta $=30 \mathrm{deg}$, phi $=90 \mathrm{deg}$ geometry 


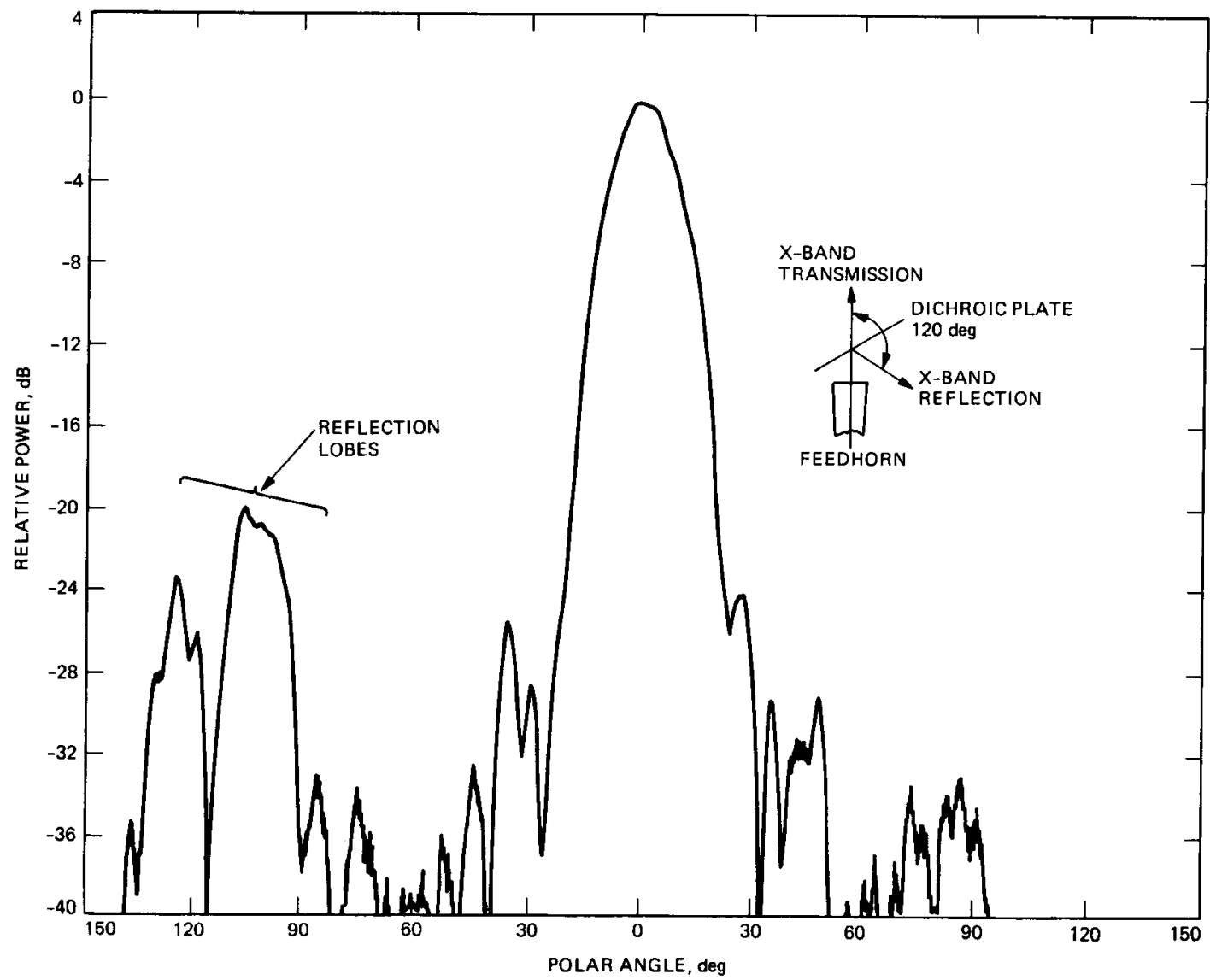

Fig. 22. H-plane pattern for X-band horn plus dichroic Plate \#1 at $7168 \mathrm{MHz}$ with plate holder flopped $90 \mathrm{deg}$ on its side; plate is in the theta $=\mathbf{3 0} \mathrm{deg}$, phi $=90 \mathrm{deg}$ geometry 


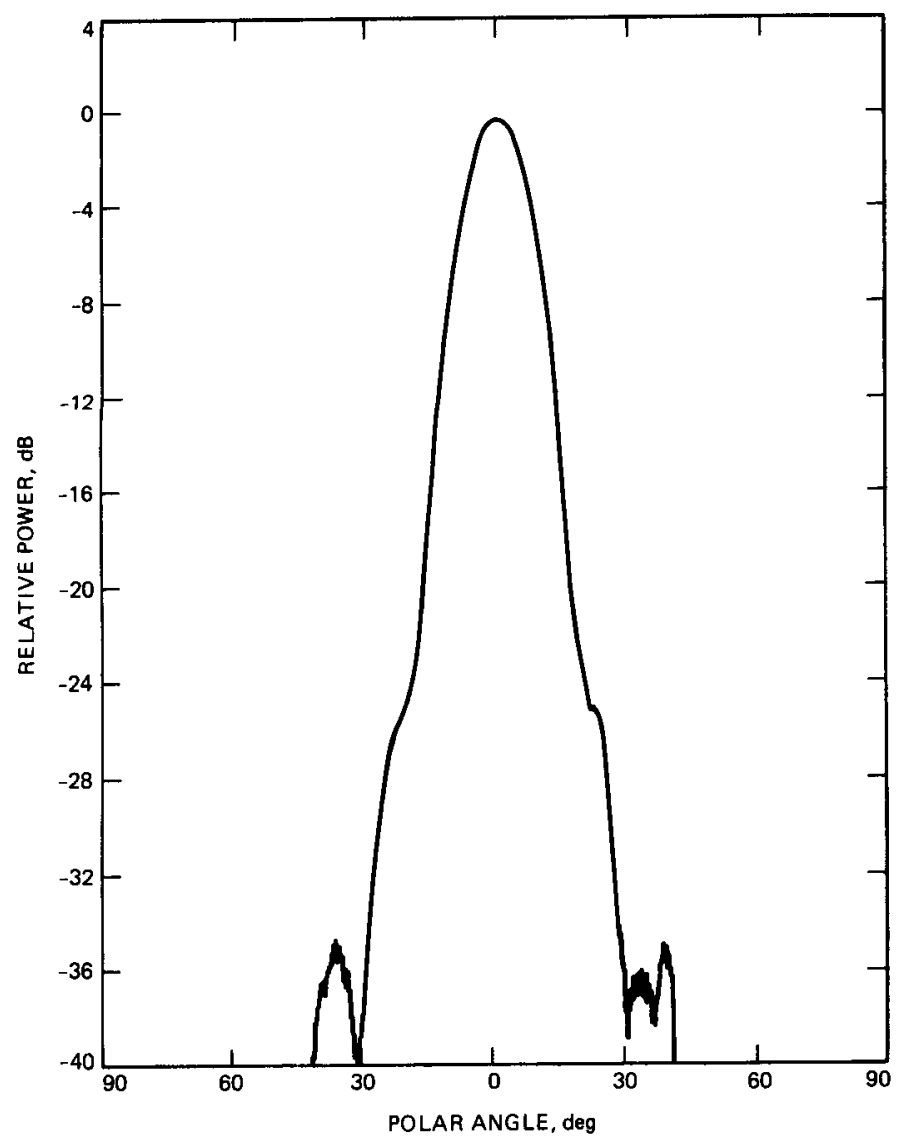

Fig. 23. E-plane pattern for X-band horn only at $8425 \mathrm{MHz}$

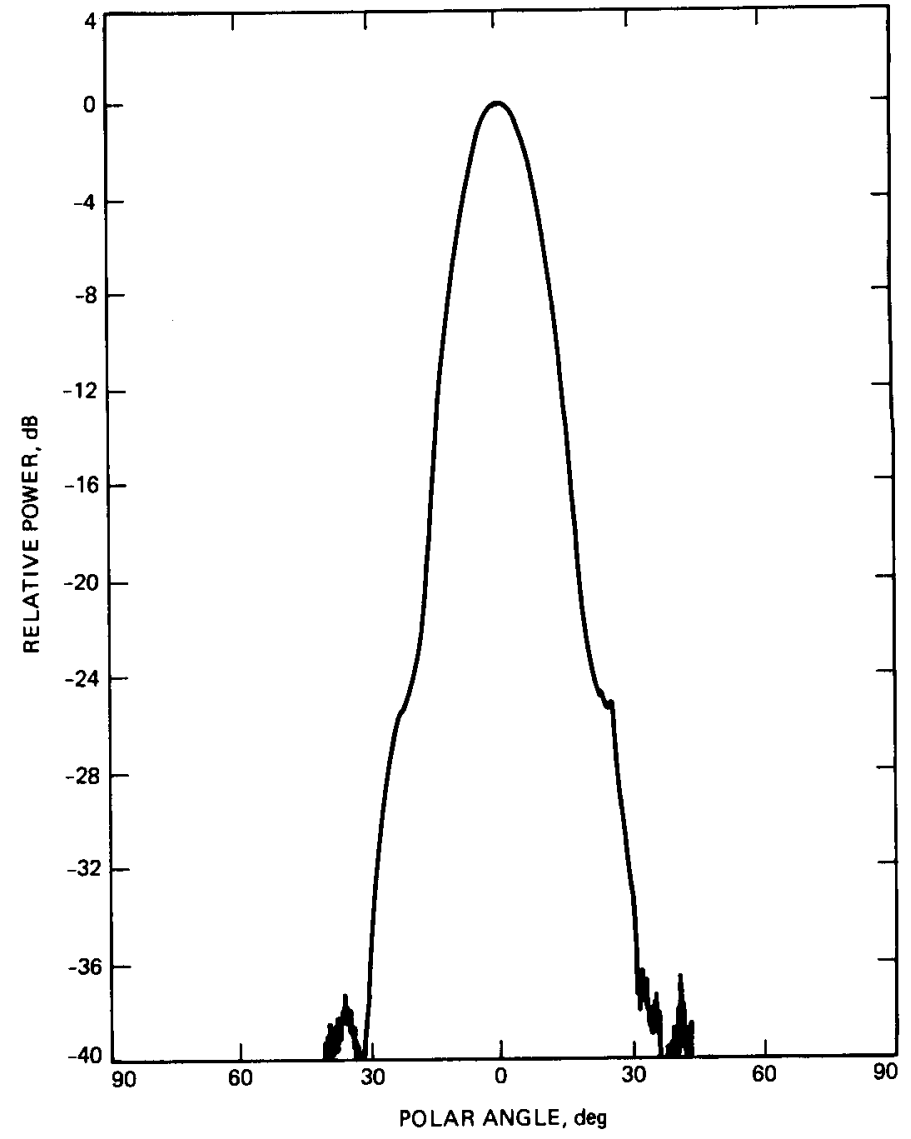

Fig. 24. H-plane pattem for $X$-band hom only at $8425 \mathrm{MHz}$ 


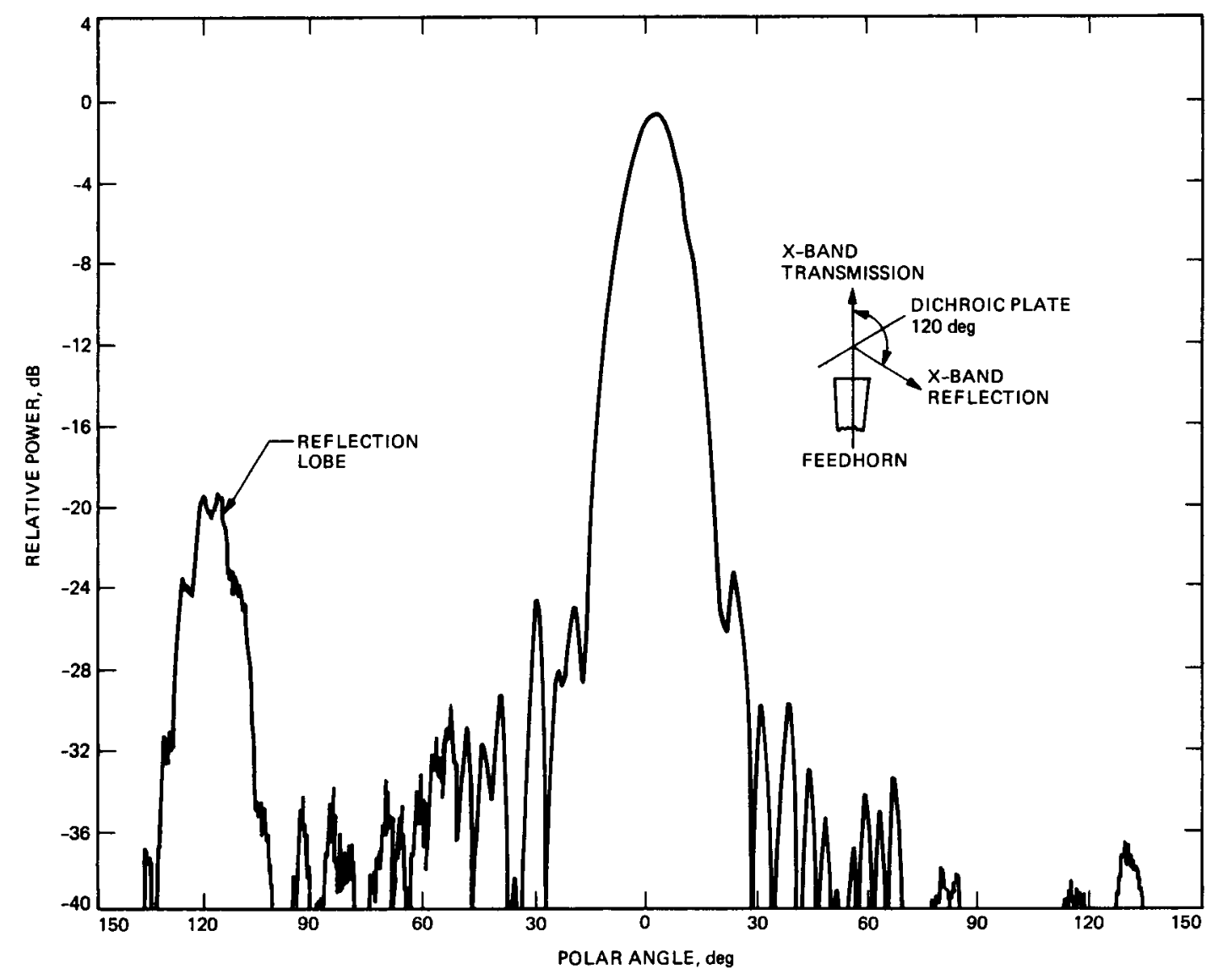

Fig. 25. E-plane pattern for $X$-band horn plus dichroic Plate \#1 at $8425 \mathrm{MHz}$ with plate holder flopped 90 deg on its side; plate is in the theta $=30 \mathrm{deg}, \mathrm{phi}=90 \mathrm{deg}$ geometry 


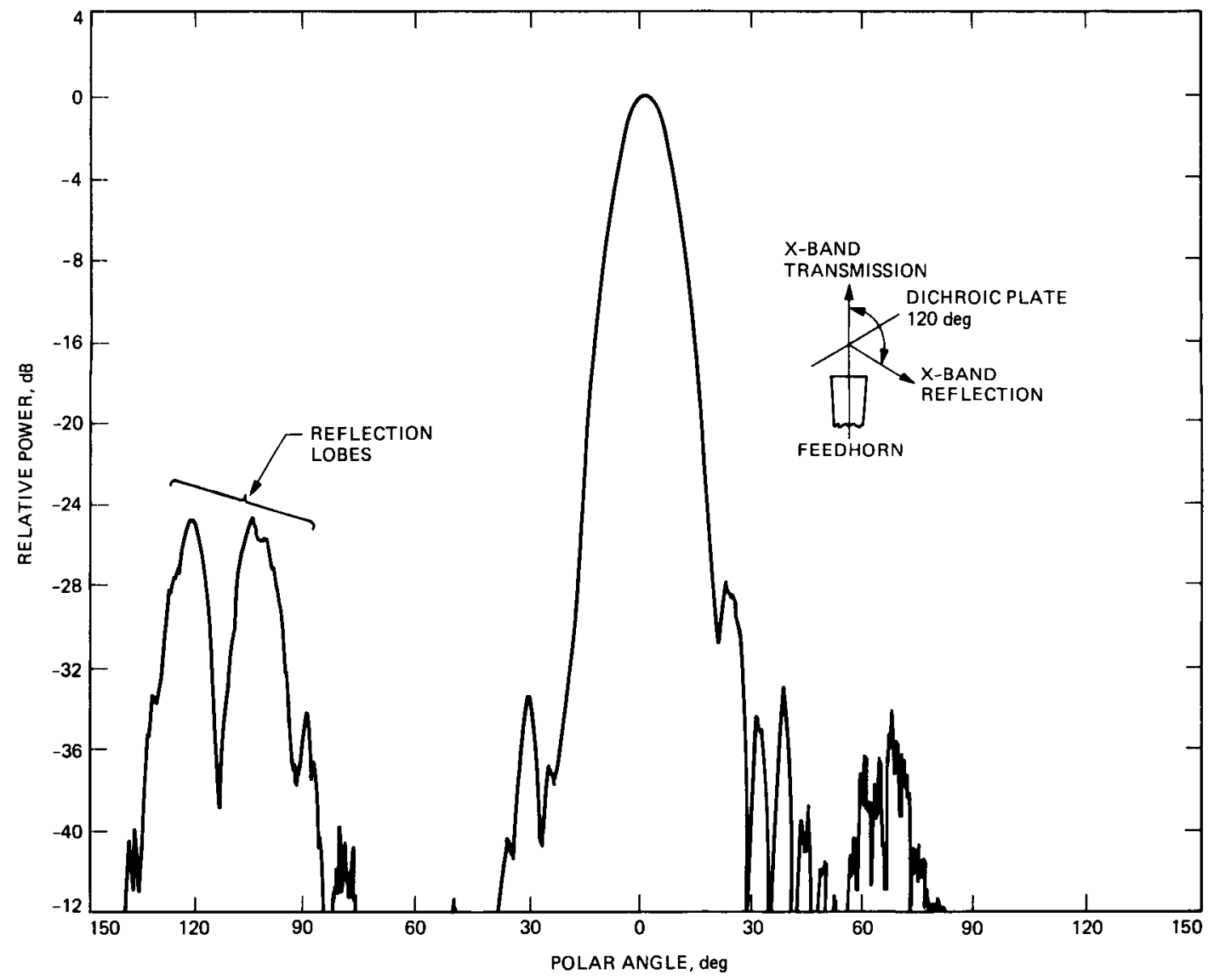

Fig. 26. H-plane pattern for X-band horn plus dichroic Plate \#1 at $8425 \mathrm{MHz}$ with plate holder flopped $90 \mathrm{deg}$ on its side; plate is in the theta $=\mathbf{3 0} \mathrm{deg}$, phi $=\mathbf{9 0}$ deg geometry 